\title{
Periodicities of T-systems and Y-systems, Dilogarithm Identities, and Cluster Algebras I: Type $B_{r}$
}

\author{
by \\ Rei Inoue, Osamu Iyama, Bernhard Keller, Atsuo Kuniba and \\ Tomoki NAKANISHI
}

\begin{abstract}
We prove the periodicities of the restricted T-systems and Y-systems associated with the quantum affine algebra of type $B_{r}$ at any level. We also prove the dilogarithm identities for the Y-systems of type $B_{r}$ at any level. Our proof is based on the tropical Y-systems and the categorification of the cluster algebra associated with any skew-symmetric matrix by Plamondon. Using this new method, we also give an alternative and simplified proof of the periodicities of the T-systems and Y-systems associated with pairs of simply laced Dynkin diagrams.
\end{abstract}

2010 Mathematics Subject Classification: Primary 13F60; Secondary 17B37.

Keywords: T-systems, Y-systems, cluster algebra, dilogarithm.

1 Main results 2

\section{Contents}

2 Cluster algebraic formulation 8

3 Tropical Y-system at level 219

4 Tropical Y-systems at higher levels 27

5 2-Calabi-Yau realization and periodicity 31

6 Dilogarithm identities 36

7 Alternative proof of periodicities of T-systems and Y-systems of simply laced type 39 References 40

Communicated by H. Nakajima. Received April 8, 2010.

R. Inoue: Faculty of Pharmaceutical Sciences, Suzuka University of Medical Science, Suzuka, 513-8670, Japan

e-mail: reiiy@math.s.chiba-u.ac.jp

O. Iyama: Graduate School of Mathematics, Nagoya University, Nagoya, 464-8604, Japan;

e-mail: iyama@math.nagoya-u.ac.jp

B. Keller: Université Paris Diderot - Paris 7, UFR de Mathématiques, Institut de Mathématiques de Jussieu, UMR 7586 du CNRS, Case 7012, 2, place Jussieu, 75251 Paris Cedex 05, France; e-mail: keller@math.jussieu.fr

A. Kuniba: Institute of Physics, University of Tokyo, Tokyo, 153-8902, Japan;

e-mail: atsuo@gokutan.c.u-tokyo.ac.jp

T. Nakanishi: Graduate School of Mathematics, Nagoya University, Nagoya, 464-8604, Japan; e-mail: nakanisi@math.nagoya-u.ac.jp 


\section{$\S 1$. Main results}

\section{$\S 1.1$. Background}

The T-systems and Y-systems are systems of algebraic relations originally associated with quantum affine algebras [KNS2, Nkj, Her1], or more generally, with the quantum affinizations of a wide class of quantum Kac-Moody algebras [Her2, KNS3].

On the other hand, these systems also appear naturally in cluster algebras [FZ2, FZ3]. This identification has provided several fruitful results. The periodicities of Y-systems were proved by [FZ2] for any simply laced type at level 2 (in our terminology). Here by 'simply laced' we mean the Y-systems associated with the quantum affine algebras of simply laced type. The periodicities of Y-systems were further proved by [Ke1, Ke2] for any simply laced type at any level, by the combination with the cluster category method. Using the method of [Ke1, Ke2], the periodicities of T-systems were also proved by [IIKNS] for any simply laced type at any level. Closely related to the Y-systems, the dilogarithm identities were proved by [C] for any simply laced type at level 2 based on the result of [FZ2], and further by [Nkn] for any simply laced type and any level. So far, however, all these systematic treatments were limited to the simply laced case only, since the above methods are not straightforwardly applicable to the non-simply laced case.

In this paper and the subsequent one [IIKKN], we prove the periodicities of Tsystems and Y-systems, and also the dilogarithm identities, in the non-simply laced case using the cluster algebra/cluster category method with suitable modifications in comparison to the method used in the simply laced case. We remark that the non-simply laced systems here are different from those arising from cluster algebras and studied in [FZ2, FZ3].

As is often the case in the non-simply laced setting, each type requires some nonuniform, 'customized' treatment. So, in this paper, we concentrate on type $B_{r}$ and highlight the underlying common method. Then, in [IIKKN], types $C_{r}, F_{4}$, and $G_{2}$ will be treated with emphasis on the special features of each case.

\section{$\S 1.2$. Restricted T-systems and Y-systems of type $B_{r}$}

Let $B_{r}$ be the Dynkin diagram of type $B$ with rank $r$, and $I=\{1, \ldots, r\}$ be the enumeration of the vertices of $B_{r}$ :

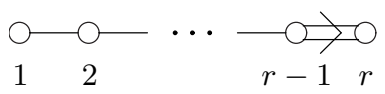

Let $h=2 r$ and $h^{\vee}=2 r-1$ be the Coxeter number and the dual Coxeter number 
of $B_{r}$, respectively. We set

$$
t_{a}= \begin{cases}1, & a=1, \ldots, r-1, \\ 2, & a=r .\end{cases}
$$

For a given integer $\ell \geq 2$, we define

$$
\mathcal{I}_{\ell}=\mathcal{I}_{\ell}\left(B_{r}\right):=\left\{(a, m, u) \mid a \in I ; m=1, \ldots, t_{a} \ell-1 ; u \in \frac{1}{2} \mathbb{Z}\right\} .
$$

Definition 1.1 ([KNS2]). Fix an integer $\ell \geq 2$. The level $\ell$ restricted $T$-system $\mathbb{T}_{\ell}\left(B_{r}\right)$ of type $B_{r}$ (with the unit boundary condition) is the following system of relations (1.3) for a family of variables $T_{\ell}=\left\{T_{m}^{(a)}(u) \mid(a, m, u) \in \mathcal{I}_{\ell}\right\}$, where $T_{m}^{(0)}(u)=T_{0}^{(a)}(u)=1$, and furthermore $T_{t_{a} \ell}^{(a)}(u)=1$ (the unit boundary condition) if they occur in the right hand sides of the relations.

(Here and throughout the paper, $2 m$ (resp. $2 m+1$ ) in the left hand sides, for example, represents elements $2,4, \ldots(\operatorname{resp} .1,3, \ldots)$.)

$$
\begin{aligned}
& T_{m}^{(a)}(u-1) T_{m}^{(a)}(u+1)= T_{m-1}^{(a)}(u) T_{m+1}^{(a)}(u)+T_{m}^{(a-1)}(u) T_{m}^{(a+1)}(u) \\
& \quad(1 \leq a \leq r-2), \\
& T_{m}^{(r-1)}(u-1) T_{m}^{(r-1)}(u+1)= T_{m-1}^{(r-1)}(u) T_{m+1}^{(r-1)}(u)+T_{m}^{(r-2)}(u) T_{2 m}^{(r)}(u), \\
& T_{2 m}^{(r)}\left(u-\frac{1}{2}\right) T_{2 m}^{(r)}\left(u+\frac{1}{2}\right)= T_{2 m-1}^{(r)}(u) T_{2 m+1}^{(r)}(u) \\
&+T_{m}^{(r-1)}\left(u-\frac{1}{2}\right) T_{m}^{(r-1)}\left(u+\frac{1}{2}\right), \\
& T_{2 m+1}^{(r)}\left(u-\frac{1}{2}\right) T_{2 m+1}^{(r)}\left(u+\frac{1}{2}\right)= T_{2 m}^{(r)}(u) T_{2 m+2}^{(r)}(u)+T_{m}^{(r-1)}(u) T_{m+1}^{(r-1)}(u) .
\end{aligned}
$$

Definition $1.2([\mathrm{KN}])$. Fix an integer $\ell \geq 2$. The level $\ell$ restricted $Y$-system $\mathbb{Y}_{\ell}\left(B_{r}\right)$ of type $B_{r}$ is the following system of relations (1.4) for a family of variables $Y_{\ell}=\left\{Y_{m}^{(a)}(u) \mid(a, m, u) \in \mathcal{I}_{\ell}\right\}$, where $Y_{m}^{(0)}(u)=Y_{0}^{(a)}(u)^{-1}=Y_{t_{a} \ell}^{(a)}(u)^{-1}=0$ if they occur in the right hand sides of the relations:

$$
\begin{aligned}
Y_{m}^{(a)}(u-1) Y_{m}^{(a)}(u+1) & =\frac{\left(1+Y_{m}^{(a-1)}(u)\right)\left(1+Y_{m}^{(a+1)}(u)\right)}{\left(1+Y_{m-1}^{(a)}(u)^{-1}\right)\left(1+Y_{m+1}^{(a)}(u)^{-1}\right)} \\
(1 \leq a \leq r-2), & \left(1+Y_{m}^{(r-2)}(u)\right)\left(1+Y_{2 m-1}^{(r)}(u)\right)\left(1+Y_{2 m+1}^{(r)}(u)\right) \\
Y_{m}^{(r-1)}(u-1) Y_{m}^{(r-1)}(u+1) & =\frac{\left(1+Y_{2 m}^{(r)}\left(u-\frac{1}{2}\right)\right)\left(1+Y_{2 m}^{(r)}\left(u+\frac{1}{2}\right)\right)}{\left(1+Y_{m-1}^{(r-1)}(u)^{-1}\right)\left(1+Y_{m+1}^{(r-1)}(u)^{-1}\right)}, \\
Y_{2 m}^{(r)}\left(u-\frac{1}{2}\right) Y_{2 m}^{(r)}\left(u+\frac{1}{2}\right) & =\frac{1+Y_{m}^{(r-1)}(u)}{\left(1+Y_{2 m-1}^{(r)}(u)^{-1}\right)\left(1+Y_{2 m+1}^{(r)}(u)^{-1}\right)} \\
Y_{2 m+1}^{(r)}\left(u-\frac{1}{2}\right) Y_{2 m+1}^{(r)}\left(u+\frac{1}{2}\right) & =\frac{1}{\left(1+Y_{2 m}^{(r)}(u)^{-1}\right)\left(1+Y_{2 m+2}^{(r)}(u)^{-1}\right)} .
\end{aligned}
$$


Let us write (1.3) in a unified manner

$$
\begin{aligned}
T_{m}^{(a)}\left(u-\frac{1}{t_{a}}\right) T_{m}^{(a)} & \left(u+\frac{1}{t_{a}}\right) \\
& =T_{m-1}^{(a)}(u) T_{m+1}^{(a)}(u)+\prod_{(b, k, v) \in \mathcal{I}_{\ell}} T_{k}^{(b)}(v)^{G(b, k, v ; a, m, u)}
\end{aligned}
$$

Define the transposition ${ }^{t} G(b, k, v ; a, m, u)=G(a, m, u ; b, k, v)$. Then we have

$$
Y_{m}^{(a)}\left(u-\frac{1}{t_{a}}\right) Y_{m}^{(a)}\left(u+\frac{1}{t_{a}}\right)=\frac{\prod_{(b, k, v) \in \mathcal{I}_{\ell}}\left(1+Y_{k}^{(b)}(v)\right)^{t} G(b, k, v ; a, m, u)}{\left(1+Y_{m-1}^{(a)}(u)^{-1}\right)\left(1+Y_{m+1}^{(a)}(u)^{-1}\right)} .
$$

See [IIKNS, KNS3] and references therein for the background of these systems.

\section{§1.3. Periodicities}

Definition 1.3. Let $\mathcal{T}_{\ell}\left(B_{r}\right)$ be the commutative ring over $\mathbb{Z}$ with identity element, with generators $T_{m}^{(a)}(u)^{ \pm 1}\left((a, m, u) \in \mathcal{I}_{\ell}\right)$ and relations $\mathbb{T}_{\ell}\left(B_{r}\right)$ together with $T_{m}^{(a)}(u) T_{m}^{(a)}(u)^{-1}=1$. Let $\mathcal{T}_{\ell}^{\circ}\left(B_{r}\right)$ be the subring of $\mathcal{T}_{\ell}\left(B_{r}\right)$ generated by $T_{m}^{(a)}(u)$ $\left((a, m, u) \in \mathcal{I}_{\ell}\right)$.

A semifield $(\mathbb{P}, \oplus)$ is an abelian multiplicative group $\mathbb{P}$ endowed with addition $\oplus$ which is commutative, associative, and distributive with respect to multiplication in $\mathbb{P}[\mathrm{FZ} 3, \mathrm{HW}]$.

Definition 1.4. Let $y_{\ell}\left(B_{r}\right)$ be the semifield with generators $Y_{m}^{(a)}(u)((a, m, u)$ $\left.\in \mathcal{I}_{\ell}\right)$ and relations $\mathbb{Y}_{\ell}\left(B_{r}\right)$. Let $y_{\ell}^{\circ}\left(B_{r}\right)$ be the multiplicative subgroup of $y_{\ell}\left(B_{r}\right)$ generated by $Y_{m}^{(a)}(u), 1+Y_{m}^{(a)}(u)\left((a, m, u) \in \mathcal{I}_{\ell}\right)$. (Here we use + instead of $\oplus$ for simplicity.)

The first main result of the paper concerns the periodicities of the T-systems and Y-systems.

Theorem 1.5 (Conjectured in [IIKNS]). The following relations hold in $\mathcal{T}_{\ell}^{\circ}\left(B_{r}\right)$ :

(i) Half periodicity: $T_{m}^{(a)}\left(u+h^{\vee}+\ell\right)=T_{t_{a} \ell-m}^{(a)}(u)$.

(ii) Full periodicity: $T_{m}^{(a)}\left(u+2\left(h^{\vee}+\ell\right)\right)=T_{m}^{(a)}(u)$.

Theorem 1.6 (Conjectured in [KNS2]). The following relations hold in $y_{\ell}^{\circ}\left(B_{r}\right)$ :

(i) Half periodicity: $Y_{m}^{(a)}\left(u+h^{\vee}+\ell\right)=Y_{t_{a} \ell-m}^{(a)}(u)$.

(ii) Full periodicity: $Y_{m}^{(a)}\left(u+2\left(h^{\vee}+\ell\right)\right)=Y_{m}^{(a)}(u)$. 


\section{§1.4. Dilogarithm identities}

Let $L(x)$ be the Rogers dilogarithm function [L, Ki2, Zag]:

$$
L(x)=-\frac{1}{2} \int_{0}^{x}\left\{\frac{\log (1-y)}{y}+\frac{\log y}{1-y}\right\} d y \quad(0 \leq x \leq 1) .
$$

It is well known that

$$
\begin{gathered}
L(0)=0, \quad L(1)=\pi^{2} / 6, \\
L(x)+L(1-x)=\pi^{2} / 6 \quad(0 \leq x \leq 1) .
\end{gathered}
$$

We introduce the constant version of the Y-system.

Definition 1.7. Fix an integer $\ell \geq 2$. The level $\ell$ restricted constant $Y$-system $\mathbb{Y}_{\ell}^{\mathrm{c}}\left(B_{r}\right)$ of type $B_{r}$ is the following system of relations (1.10) for a family of variables $Y_{\ell}^{\mathrm{c}}=\left\{Y_{m}^{(a)} \mid a \in I ; m=1, \ldots, t_{a} \ell-1\right\}$, where $Y_{m}^{(0)}=Y_{0}^{(a)}-1=Y_{t_{a} \ell}^{(a)}-1=0$ if they occur in the right hand sides of the relations:

$$
\begin{aligned}
\left(Y_{m}^{(a)}\right)^{2} & =\frac{\left(1+Y_{m}^{(a-1)}\right)\left(1+Y_{m}^{(a+1)}\right)}{\left(1+Y_{m-1}^{(a)}-1\right)\left(1+Y_{m+1}^{(a)}-1\right)} \quad(1 \leq a \leq r-2) \\
\left(Y_{m}^{(r-1)}\right)^{2} & =\frac{\left(1+Y_{m}^{(r-2)}\right)\left(1+Y_{2 m-1}^{(r)}\right)\left(1+Y_{2 m}^{(r)}\right)^{2}\left(1+Y_{2 m+1}^{(r)}\right)}{\left(1+Y_{m-1}^{(r-1)}-1\right)\left(1+Y_{m+1}^{(r-1)}-1\right)} \\
\left(Y_{2 m}^{(r)}\right)^{2} & =\frac{1+Y_{m}^{(r-1)}}{\left(1+Y_{2 m-1}^{(r)}-1\right)\left(1+Y_{2 m+1}^{(r)}-1\right)}, \\
\left(Y_{2 m+1}^{(r)}\right)^{2} & =\frac{1}{\left(1+Y_{2 m}^{(r)}-1\right)\left(1+Y_{2 m+2}^{(r)}-1\right)}
\end{aligned}
$$

Proposition 1.8. There exists a unique positive real solution of $\mathbb{Y}_{\ell}^{\mathrm{c}}\left(B_{r}\right)$.

Proof. Set $f_{m}^{(a)}=Y_{m}^{(a)} /\left(1+Y_{m}^{(a)}\right)$. Then (1.10) is equivalent to the system of equations [KNS2, Eq. (B.28)]

$$
f_{m}^{(a)}=\prod_{(b, k)}\left(1-f_{k}^{(b)}\right)^{K_{a b}^{m k}}, \quad K_{a b}^{m k}=\left(\alpha_{a} \mid \alpha_{b}\right)\left(\min \left(t_{b} m, t_{a} k\right)-m k / \ell\right),
$$

where $\left(\alpha_{a} \mid \alpha_{b}\right)$ is the invariant bilinear form for the simple Lie algebra of type $B_{r}$ with normalization $\left(\alpha_{a} \mid \alpha_{a}\right)=2$ for a long root $\alpha_{a}$. By elementary transformations, one can show that every principal minor of the matrix $K$ is positive. Therefore, $K$ is positive definite. Also, it is clear that $K$ is symmetric. Therefore, the theorem of [NK, Section 1] is applicable. 
The second main result of the paper is the dilogarithm identities conjectured by Kirillov [Ki1, Eq. (7)], and corrected by Kuniba [Ku, Eqs. (A.1a), (A.1c)]:

Theorem 1.9 (Dilogarithm identities). Suppose that a family of positive real numbers $\left\{Y_{m}^{(a)} \mid a \in I ; m=1, \ldots, t_{a} \ell-1\right\}$ satisfies (1.10). Then

$$
\frac{6}{\pi^{2}} \sum_{a \in I} \sum_{m=1}^{t_{a} \ell-1} L\left(\frac{Y_{m}^{(a)}}{1+Y_{m}^{(a)}}\right)=\frac{\ell \operatorname{dim} \mathfrak{g}}{h^{\vee}+\ell}-r,
$$

where $\mathfrak{g}$ is the simple Lie algebra of type $B_{r}$.

The rational number of the first term on the right hand side of (1.12) is the central charge of the Wess-Zumino-Witten conformal field theory of type $B_{r}$ with level $\ell$. The rational number on the right hand side of (1.12) itself is also the central charge of the parafermion conformal field theory of type $B_{r}$ with level $\ell$. See [KNS1, Ki2, Nah, Zag] for more background of (1.12).

By the well-known formula $\operatorname{dim} \mathfrak{g}=r(h+1)$, the right hand side of $(1.12)$ is equal to

$$
\frac{r\left(\ell h-h^{\vee}\right)}{h^{\vee}+\ell} .
$$

In fact, we prove a functional generalization of Theorem 1.9, following the ideas of [GT, FS, C, Nkn].

Theorem 1.10 (Functional dilogarithm identities). Suppose that a family of positive real numbers $\left\{Y_{m}^{(a)}(u) \mid(a, m, u) \in \mathcal{I}_{\ell}\right\}$ satisfies $\mathbb{Y}_{\ell}\left(B_{r}\right)$. Then

$$
\begin{aligned}
& \frac{6}{\pi^{2}} \sum_{\substack{(a, m, u) \in \mathcal{I}_{\ell} \\
0 \leq u<2\left(h^{\vee}+\ell\right)}} L\left(\frac{Y_{m}^{(a)}(u)}{1+Y_{m}^{(a)}(u)}\right)=4 r\left(\ell h-h^{\vee}\right)=4 r(2 r \ell-2 r+1), \\
& \frac{6}{\pi^{2}} \sum_{\substack{(a, m, u) \in \mathcal{I}_{\ell} \\
0 \leq u<2\left(h^{\vee}+\ell\right)}} L\left(\frac{1}{1+Y_{m}^{(a)}(u)}\right)=4 \ell(r \ell+\ell-1) .
\end{aligned}
$$

The two identities (1.14) and (1.15) are equivalent to each other due to (1.9), since the sum of their right hand sides is equal to $4\left(h^{\vee}+\ell\right)(r \ell+\ell-r)$, which is the total number of $(a, m, u) \in I_{\ell}$ with $0 \leq u<2\left(h^{\vee}+\ell\right)$.

It is clear that Theorem 1.9 follows from Theorem 1.10 by considering a constant solution $Y_{m}^{(a)}=Y_{m}^{(a)}(u)$ of $\mathbb{Y}_{\ell}\left(B_{r}\right)$ in the variable $u$.

\section{$\S 1.5$. Outline of the method and contents}

Let us briefly explain the idea of our proof of the main results, Theorems 1.5, 1.6, and 1.10 . 
To start with, we identify the T-systems and Y-systems, $\mathbb{T}_{\ell}\left(B_{r}\right)$ and $\mathbb{Y}_{\ell}\left(B_{r}\right)$ in Definitions 1.1 and 1.2, as systems of relations for cluster variables and coefficients of a cluster algebra, respectively. This procedure is mostly parallel to the simply laced case [FZ2, FZ3, Ke1, DiK, HL, IIKNS, KNS3], but necessarily more complicated. For example, unlike the simply laced case, the composite mutation which generates the translation of the spectral parameter $u$ is neither of bipartite type nor obviously related to the Coxeter element of a certain Weyl group. This is not a serious problem, though. A real problem is that the arising quiver $Q_{\ell}\left(B_{r}\right)$ for the cluster algebra, which is seen in Figure 1 of Section 2.3, is not a familiar one in the representation theory of quivers; in particular, we have no known or obvious periodicity result. This is the main obstacle to a straightforward application of the method in [Ke1, IIKNS, Ke2], where the periodicities of the T-systems and Y-systems in the simply laced case were derived from the periodicity in the corresponding cluster category.

The key to bypassing this obstacle is to consider the tropical Y-system. The tropical Y-system is the tropicalization of the Y-system, or more generally, of the exchange relations of the coefficients in the tropical semifield (called principal coefficients in [FZ3]) of a given cluster algebra. In fact, it was already used by Fomin-Zelevinsky [FZ2] as a main tool in the proof of the periodicity of the Ysystems in the simply laced case at level 2. In addition, we make two crucial observations.

Observation 1. The periodicities of cluster variables and coefficients follow from the periodicity of principal coefficients.

See Theorem 5.1 for a precise statement. This claim was essentially conjectured by Fomin-Zelevinsky [FZ3, Conjecture 4.7]. We prove the claim for the cluster algebra associated with any skew-symmetric matrix, or equivalently, with any quiver. To prove it, we use the recent result by Plamondon [Pl1, Pl2] on the categorification of the cluster algebra associated with an arbitrary quiver. It is so far the most general formulation of the categorification by 2-Calabi-Yau categories recently developed by various authors in particular cases (see $[\mathrm{A}]$ and the references therein). Since each principal coefficient tuple carries complete information on the corresponding object in the category through index, the periodicity of principal coefficients implies the same periodicity of objects in the category. Therefore, it also implies the same periodicities of cluster variables and coefficients by categorification.

Observation 2. The tropical $Y$-system for $\mathbb{Y}_{\ell}\left(B_{r}\right)$ has a remarkable 'factorization property' so that its periodicity can be directly verified. 
This factorization property was first noticed by $[\mathrm{Nkn}]$ in the proof of the dilogarithm identities in the simply laced case. It roughly means that the tropical Y-system at a higher level splits into level 2 pieces and type $A$ pieces. Moreover, each piece can be described in terms of the piecewise-linear analogue of the simple reflections of a certain Weyl group introduced by [FZ2]. Therefore, the periodicity is tractable.

Combining these two observations, we obtain the desired periodicities in Theorems 1.5 and 1.6.

The tropical Y-system plays a central role not only in the periodicity but also in the dilogarithm identity. The following observation was made in [ $\mathrm{Nkn}]$ in the simply laced case.

Observation 3. The dilogarithm identity reduces to the positivity/negativity property of the tropical $Y$-system for $\mathbb{Y}_{\ell}\left(B_{r}\right)$.

Briefly, "the tropical Y-system knows everything".

The organization of the paper is as follows. In Section 2, we introduce a quiver $Q_{\ell}\left(B_{r}\right)$ and identify the T-systems and Y-systems with systems of relations for cluster variables and coefficients of the cluster algebra associated with $Q_{\ell}\left(B_{r}\right)$ (Theorems 2.7 and 2.10). In Section 3, we study the tropical Y-system at level 2 and derive the periodicity and the positivity/negativity property (Proposition 3.2). In Section 4, we study the tropical Y-system at higher levels and show the factorization property. As a result, we obtain the periodicity (Theorems 4.2) and the positivity/negativity property (Theorem 4.3). In Section 5, based on the result by Plamondon, we present a general theorem giving Observation 1 above (Theorem 5.1) for the cluster algebra associated with any skew-symmetric matrix. As corollaries, we obtain the periodicities of T-systems and Y-systems in Theorems 1.5 and 1.6. In Section 6, using Theorem 4.3, we prove the dilogarithm identity of Theorem 1.10. In Section 7, as feedback from the newly introduced method, we give an alternative and simplified proof of the periodicities of the T-systems and Y-systems associated with pairs of simply laced Dynkin diagrams, which were formerly proved in [Ke1, IIKNS, Ke2].

\section{$\S 2$. Cluster algebraic formulation}

The systems $\mathbb{T}_{\ell}\left(B_{r}\right)$ and $\mathbb{Y}_{\ell}\left(B_{r}\right)$ are naturally identified with systems of relations for cluster variables and coefficients, respectively, of a certain cluster algebra. They are mostly parallel to the simply laced case [FZ2, FZ3, Ke1, DiK, HL, IIKNS, KNS3], but necessarily more complicated. 


\section{§2.1. Definitions for cluster algebras}

Here we collect basic definitions for cluster algebras to fix the conventions and notation, mainly following [FZ3]. For further necessary definitions and information on cluster algebras, see [FZ3].

Let $I$ be a finite index set throughout this subsection.

(i) Semifield. A semifield $(\mathbb{P}, \oplus, \cdot)$ is an abelian multiplicative group endowed with addition $\oplus$ which is commutative, associative, and distributive with respect to multiplication in $\mathbb{P}$. The following three examples are relevant in this paper.

(a) Trivial semifield. The trivial semifield $\mathbf{1}=\{1\}$ consists of the multiplicative identity element 1 with $1 \oplus 1=1$.

(b) Universal semifield. For an $I$-tuple of variables $y=\left(y_{i}\right)_{i \in I}$, the universal semifield $\mathbb{Q}_{\mathrm{sf}}(y)$ consists of all the rational functions of the form $P(y) / Q(y)$ (subtraction-free rational expressions), where $P(y)$ and $Q(y)$ are the nonzero polynomials in $y_{i}$ 's with nonnegative integer coefficients. Multiplication and addition are given by the usual operations on rational functions.

(c) Tropical semifield. For an $I$-tuple of variables $y=\left(y_{i}\right)_{i \in I}$, the tropical semifield $\operatorname{Trop}(y)$ is the abelian multiplicative group freely generated by the variables $y_{i}$ endowed with addition $\oplus$ defined by

$$
\prod_{i} y_{i}^{a_{i}} \oplus \prod_{i} y_{i}^{b_{i}}=\prod_{i} y_{i}^{\min \left(a_{i}, b_{i}\right)}
$$

(ii) Mutations of matrices and quivers. An integer matrix $B=\left(B_{i j}\right)_{i, j \in I}$ is skew-symmetrizable if there is a diagonal matrix $D=\operatorname{diag}\left(d_{i}\right)_{i \in I}$ with $d_{i} \in \mathbb{N}$ such that $D B$ is skew-symmetric. For a skew-symmetrizable matrix $B$ and $k \in I$, another matrix $B^{\prime}=\mu_{k}(B)$, called the mutation of $B$ at $k$, is defined by

$$
B_{i j}^{\prime}= \begin{cases}-B_{i j}, & i=k \text { or } j=k \\ B_{i j}+\frac{1}{2}\left(\left|B_{i k}\right| B_{k j}+B_{i k}\left|B_{k j}\right|\right), & \text { otherwise. }\end{cases}
$$

The matrix $\mu_{k}(B)$ is also skew-symmetrizable.

It is standard to represent a skew-symmetric (integer) matrix $B=\left(B_{i j}\right)_{i, j \in I}$ by a quiver $Q$ without loops or 2-cycles. The set of vertices of $Q$ is given by $I$, and we put $B_{i j}$ arrows from $i$ to $j$ if $B_{i j}>0$. The mutation $Q^{\prime}=\mu_{k}(Q)$ of the quiver $Q$ is given by the following rule: For each pair of an incoming arrow $i \rightarrow k$ and an outgoing arrow $k \rightarrow j$ in $Q$, add a new arrow $i \rightarrow j$. Then, remove a maximal set of pairwise disjoint 2-cycles. Finally, reverse all arrows incident with $k$.

(iii) Exchange relation of a coefficient tuple. Let $\mathbb{P}$ be a given semifield. For an $I$-tuple $y=\left(y_{i}\right)_{i \in I}$ with $y_{i} \in \mathbb{P}$ and $k \in I$, another $I$-tuple $y^{\prime}$ is defined by the 
exchange relation

$$
y_{i}^{\prime}= \begin{cases}y_{k}^{-1}, & i=k, \\ y_{i}\left(\frac{y_{k}}{1 \oplus y_{k}}\right)^{B_{k i}}, & i \neq k, \quad B_{k i} \geq 0, \\ y_{i}\left(1 \oplus y_{k}\right)^{-B_{k i} b} & i \neq k, \quad B_{k i} \leq 0 .\end{cases}
$$

(iv) Exchange relation of a cluster. Let $\mathbb{Q P}$ be the quotient field of the group ring $\mathbb{Z P}$ of $\mathbb{P}$, and let $\mathbb{Q P}(z)$ be the rational function field of algebraically independent variables, say, $z=\left(z_{i}\right)_{i \in I}$ over $\mathbb{Q P}$. For an $I$-tuple $x=\left(x_{i}\right)_{i \in I}$ which is a free generating set of $\mathbb{Q P}(z)$ and $k \in I$, another $I$-tuple $x^{\prime}$ is defined by the exchange relation

$$
x_{i}^{\prime}= \begin{cases}x_{i}, & i \neq k, \\ \frac{y_{k} \prod_{j: B_{j k}>0} x_{j}^{B_{j k}}+\prod_{j: B_{j k}<0} x_{j}^{-B_{j k}}}{\left(1 \oplus y_{k}\right) x_{k}}, & i=k .\end{cases}
$$

(v) Seed mutation. For the above triplet $(B, x, y)$ in (ii)-(iv), which is called a seed, the mutation $\mu_{k}(B, x, y)=\left(B^{\prime}, x^{\prime}, y^{\prime}\right)$ at $k$ is defined by combining (2.2), (2.3), and (2.4).

(vi) Cluster algebra. Fix a semifield $\mathbb{P}$ and a seed (initial seed) $(B, x, y)$, where $x=\left(x_{i}\right)_{i \in I}$ are algebraically independent variables over $\mathbb{Q P}$. Starting from $(B, x, y)$, iterate mutations and collect all the seeds $\left(B^{\prime}, x^{\prime}, y^{\prime}\right)$. We call $y^{\prime}$ and $y_{i}^{\prime}$ a coefficient tuple and a coefficient, respectively. We call $x^{\prime}$ and $x_{i}^{\prime} \in \mathbb{Q P}(x)$ a cluster and a cluster variable, respectively. The cluster algebra $\mathcal{A}(B, x, y)$ with coefficients in $\mathbb{P}$ is the $\mathbb{Z P}$-subalgebra of the rational function field $\mathbb{Q P}(x)$ generated by all the cluster variables.

(vii) Cluster pattern. Let $I=\{1, \ldots, n\}$, and let $\mathbb{T}_{n}$ be the $n$-regular tree whose edges are labeled by the numbers $1, \ldots, n$. A cluster pattern is the assignment of a seed $(B(t), x(t), y(t))$ to each vertex $t \in \mathbb{T}_{n}$ so that the seeds assigned to the endpoints of any edge $t \frac{k}{t} t^{\prime}$ are obtained from each other by seed mutation at $k$. Take $t_{0} \in \mathbb{T}_{n}$ arbitrarily, and consider the cluster algebra $\mathcal{A}\left(B\left(t_{0}\right), x\left(t_{0}\right), y\left(t_{0}\right)\right)$. Then $x(t)$ and $y(t)\left(t \in \mathbb{T}_{n}\right)$ are a cluster and a coefficient tuple of $\mathcal{A}\left(B\left(t_{0}\right), x\left(t_{0}\right), y\left(t_{0}\right)\right)$.

(viii) $F$-polynomial. The cluster algebra $\mathcal{A}(B, x, y)$ with coefficients in the tropical semifield $\operatorname{Trop}(y)$ is called the cluster algebra with principal coefficients. Here, each cluster variable $x_{i}^{\prime}$ is an element in $\mathbb{Z}\left[x^{ \pm 1}, y\right]$. The $F$-polynomial $F_{i}^{\prime}(y) \in \mathbb{Z}[y]$ (for $x_{i}^{\prime}$ ) is defined as the specialization of $x_{i}^{\prime}$ with $x_{i}=1(i \in I)$. 


\section{$\S 2.2$. Parity decompositions of T-systems and Y-systems}

For a triplet $(a, m, u) \in \mathcal{I}_{\ell}$, we define the 'parity conditions' $\mathbf{P}_{+}$and $\mathbf{P}_{-}$by

$$
\begin{aligned}
& \mathbf{P}_{+}: 2 u \text { is even if } a \neq r ; m+2 u \text { is even if } a=r, \\
& \mathbf{P}_{-}: 2 u \text { is odd if } a \neq r ; m+2 u \text { is odd if } a=r .
\end{aligned}
$$

We write, for example, $(a, m, u): \mathbf{P}_{+}$if $(a, m, u)$ satisfies $\mathbf{P}_{+}$. We have $\mathcal{I}_{\ell}=$ $\mathcal{I}_{\ell+} \sqcup \mathcal{I}_{\ell-}$, where $\mathcal{I}_{\ell \varepsilon}$ is the set of all $(a, m, u): \mathbf{P}_{\varepsilon}$.

Define $\mathcal{T}_{\ell}^{\circ}\left(B_{r}\right)_{\varepsilon}(\varepsilon= \pm)$ to be the subring of $\mathcal{T}_{\ell}^{\circ}\left(B_{r}\right)$ generated by $T_{m}^{(a)}(u)$ $\left((a, m, u) \in \mathcal{I}_{\ell \varepsilon}\right)$. Then $\mathcal{T}_{\ell}^{\circ}\left(B_{r}\right)_{+} \simeq \mathcal{T}_{\ell}^{\circ}\left(B_{r}\right)_{-}$via $T_{m}^{(a)}(u) \mapsto T_{m}^{(a)}\left(u+\frac{1}{2}\right)$ and

$$
\mathcal{T}_{\ell}^{\circ}\left(B_{r}\right) \simeq \mathcal{T}_{\ell}^{\circ}\left(B_{r}\right)_{+} \otimes_{\mathbb{Z}} \mathcal{T}_{\ell}^{\circ}\left(B_{r}\right)_{-} .
$$

For a triplet $(a, m, u) \in \mathcal{I}_{\ell}$, we define other 'parity conditions' $\mathbf{P}_{+}^{\prime}$ and $\mathbf{P}_{-}^{\prime}$ by

$$
\begin{aligned}
& \mathbf{P}_{+}^{\prime}: 2 u \text { is even if } a \neq r ; m+2 u \text { is odd if } a=r, \\
& \mathbf{P}_{-}^{\prime}: 2 u \text { is odd if } a \neq r ; m+2 u \text { is even if } a=r .
\end{aligned}
$$

We have $\mathcal{I}_{\ell}=\mathcal{I}_{\ell+}^{\prime} \sqcup \mathcal{I}_{\ell-}^{\prime}$, where $\mathcal{I}_{\ell \varepsilon}^{\prime}$ is the set of all $(a, m, u): \mathbf{P}_{\varepsilon}^{\prime}$. We also have

$$
(a, m, u): \mathbf{P}_{+}^{\prime} \Leftrightarrow\left(a, m, u \pm \frac{1}{t_{a}}\right): \mathbf{P}_{+}
$$

Define $y_{\ell}^{\circ}\left(B_{r}\right)_{\varepsilon}(\varepsilon= \pm)$ to be the subgroup of $y_{\ell}^{\circ}\left(B_{r}\right)$ generated by $Y_{m}^{(a)}(u)$, $1+Y_{m}^{(a)}(u)\left((a, m, u) \in \mathcal{I}_{\ell \varepsilon}^{\prime}\right)$. Then $y_{\ell}^{\circ}\left(B_{r}\right)_{+} \simeq y_{\ell}^{\circ}\left(B_{r}\right)_{-}$by $Y_{m}^{(a)}(u) \mapsto Y_{m}^{(a)}\left(u+\frac{1}{2}\right)$, $1+Y_{m}^{(a)}(u) \mapsto 1+Y_{m}^{(a)}\left(u+\frac{1}{2}\right)$, and

$$
y_{\ell}^{\circ}\left(B_{r}\right) \simeq y_{\ell}^{\circ}\left(B_{r}\right)_{+} \times y_{\ell}^{\circ}\left(B_{r}\right)_{-} .
$$

\section{§2.3. Quiver $Q_{\ell}\left(B_{r}\right)$}

With type $B_{r}$ and $\ell \geq 2$ we associate the quiver $Q_{\ell}\left(B_{r}\right)$ by Figure 1 , where, in addition, we assign an empty or filled circle $\circ / \bullet$ and a sign $+/-$ to each vertex. Let $B_{\ell}\left(B_{r}\right)$ be the skew-symmetric matrix corresponding to $Q_{\ell}\left(B_{r}\right)$ as defined in Section 2.1(ii). (Unfortunately, there is an obvious conflict between two standard notations using symbol $B$. We hope it does not cause serious confusion to the reader.)

Let us choose the index set $\mathbf{I}$ of the vertices of $Q_{\ell}\left(B_{r}\right)$ so that $\mathbf{i}=\left(i, i^{\prime}\right) \in \mathbf{I}$ represents the vertex in the $i^{\prime}$ th row (from the bottom) and the $i$ th column (from the left). Thus, $i=1, \ldots, 2 r-1$, and $i^{\prime}=1, \ldots, \ell-1$ if $i \neq r$ and $i^{\prime}=1, \ldots, 2 \ell-1$ if $i=r$. We use the natural notation $\mathbf{I}^{\circ}$ (resp. $\mathbf{I}_{+}^{\circ}$ ) for the set of vertices $\mathbf{i}$ with property $\circ$ (resp. $\circ$ and + ), and so on. We have $\mathbf{I}=\mathbf{I}^{\circ} \sqcup \mathbf{I}^{\bullet}=\mathbf{I}_{+}^{\circ} \sqcup \mathbf{I}_{-}^{\circ} \sqcup \mathbf{I}_{+}^{\bullet} \sqcup \mathbf{I}_{-}^{\bullet}$. 

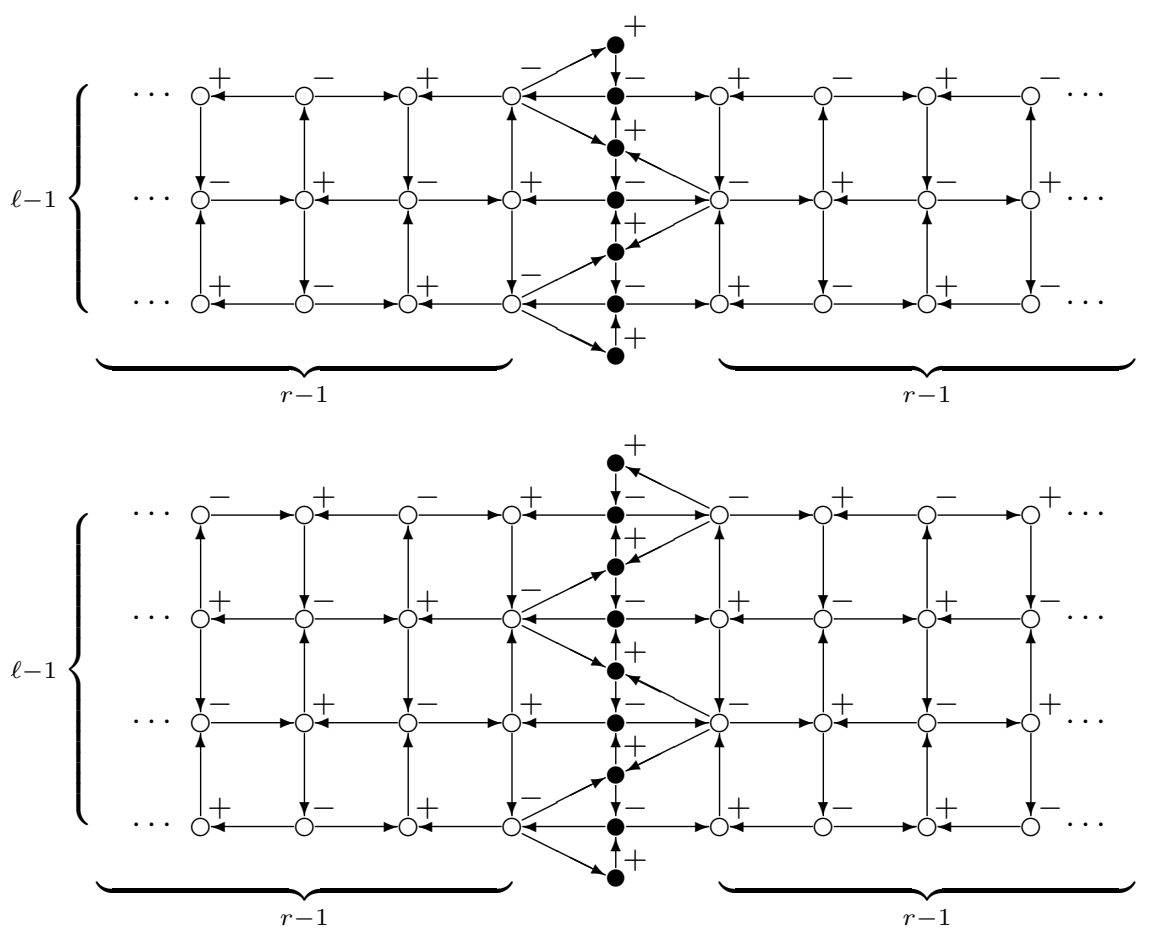

Figure 1. The quiver $Q_{\ell}\left(B_{r}\right)$ for $\ell$ even (top) and $\ell$ odd (bottom).

We define composite mutations,

$$
\mu_{+}^{\circ}=\prod_{\mathbf{i} \in \mathbf{I}_{+}^{\circ}} \mu_{\mathbf{i}}, \quad \mu_{-}^{\circ}=\prod_{\mathbf{i} \in \mathbf{I}_{-}^{\circ}} \mu_{\mathbf{i}}, \quad \mu_{+}^{\bullet}=\prod_{\mathbf{i} \in \mathbf{I}_{+}^{\bullet}} \mu_{\mathbf{i}}, \quad \mu_{-}^{\bullet}=\prod_{\mathbf{i} \in \mathbf{I}_{-}^{\bullet}} \mu_{\mathbf{i}} .
$$

Note that they do not depend on the order of the product.

Let $\boldsymbol{r}$ be the involution acting on $\mathbf{I}$ by left-right reflection. Let $\boldsymbol{\omega}$ be the involution acting on $\mathbf{I}$ by $180^{\circ}$ rotation. Let $\boldsymbol{r}\left(Q_{\ell}\left(B_{r}\right)\right)$ and $\boldsymbol{\omega}\left(Q_{\ell}\left(B_{r}\right)\right)$ denote the quivers induced from $Q_{\ell}\left(B_{r}\right)$ by $\boldsymbol{r}$ and $\boldsymbol{\omega}$, respectively. For example, if there is an arrow $\mathbf{i} \rightarrow \mathbf{j}$ in $Q_{\ell}\left(B_{r}\right)$, then there is an arrow $\boldsymbol{r}(\mathbf{i}) \rightarrow \boldsymbol{r}(\mathbf{j})$ in $\boldsymbol{r}\left(Q_{\ell}\left(B_{r}\right)\right)$. For a quiver $Q, Q^{\text {op }}$ denotes the opposite quiver.

Lemma 2.1. Let $Q=Q_{\ell}\left(B_{r}\right)$.

(i) We have a periodic sequence of mutations of quivers

$$
Q \stackrel{\mu_{+}^{\bullet} \mu_{+}^{\circ}}{\longleftrightarrow} Q^{\mathrm{op}} \stackrel{\mu_{-}^{\bullet}}{\longleftrightarrow} \boldsymbol{r}(Q) \stackrel{\mu_{+}^{\bullet} \mu_{-}^{\circ}}{\longleftrightarrow} \boldsymbol{r}(Q)^{\mathrm{op}} \stackrel{\mu_{-}^{\bullet}}{\longleftrightarrow} Q .
$$

(ii) $\boldsymbol{\omega}(Q)=Q$ if $h^{\vee}+\ell$ is even, and $\boldsymbol{\omega}(Q)=\boldsymbol{r}(Q)$ if $h^{\vee}+\ell$ is odd. 
Example 2.2. The sequence (2.13) for $Q=Q_{2}\left(B_{3}\right)$ is given below.

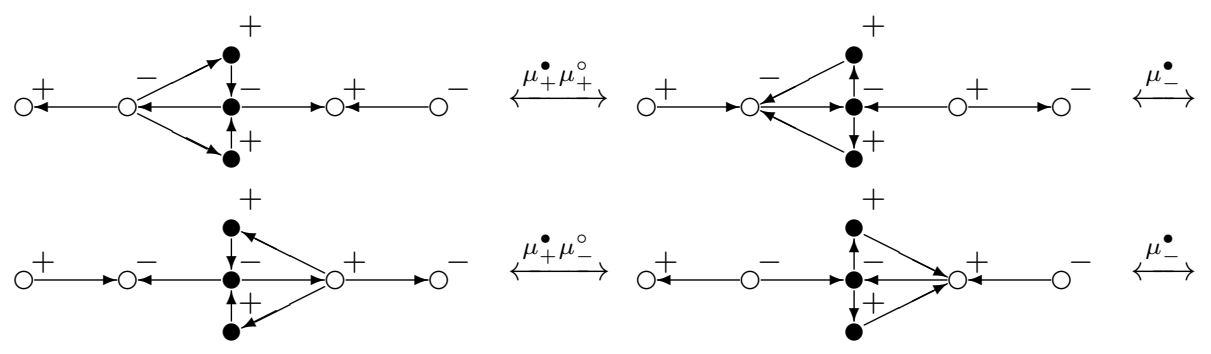

§2.4. The cluster algebra $\mathcal{A}(B, x, y)$ and the coefficient group $\mathcal{G}(B, y)$

For the matrix $B=\left(B_{\mathbf{i j}}\right)_{\mathbf{i}, \mathbf{j} \in \mathbf{I}}=B_{\ell}\left(B_{r}\right)$, let $\mathcal{A}(B, x, y)$ be the cluster algebra with coefficients in the universal semifield $\mathbb{Q}_{\mathrm{sf}}(y)$, where $(B, x, y)$ is the initial seed. (Here we use the symbol + instead of $\oplus$ in $\mathbb{Q}_{\mathrm{sf}}(y)$, since it is the ordinary addition of subtraction-free expressions of rational functions of $y$.)

For our purpose, it is natural to introduce not only the 'ring of cluster variables' but also the 'group of coefficients'.

Definition 2.3. The coefficient group $\mathcal{G}(B, y)$ associated with $\mathcal{A}(B, x, y)$ is the multiplicative subgroup of the semifield $\mathbb{Q}_{\mathrm{sf}}(y)$ generated by all the coefficients $y_{\mathbf{i}}^{\prime}$ of $\mathcal{A}(B, x, y)$ together with $1+y_{\mathbf{i}}^{\prime}$.

In view of Lemma 2.1 we set $x(0)=x, y(0)=y$ and define clusters $x(u)=$ $\left(x_{\mathbf{i}}(u)\right)_{\mathbf{i} \in \mathbf{I}}\left(u \in \frac{1}{2} \mathbb{Z}\right)$ and coefficient tuples $y(u)=\left(y_{\mathbf{i}}(u)\right)_{\mathbf{i} \in \mathbf{I}}\left(u \in \frac{1}{2} \mathbb{Z}\right)$ by the sequence of mutations

$$
\begin{aligned}
& \cdots \stackrel{\mu_{-}^{\bullet}}{\longleftrightarrow}(B, x(0), y(0)) \stackrel{\mu_{+}^{\bullet} \mu_{+}^{\circ}}{\longrightarrow}\left(-B, x\left(\frac{1}{2}\right), y\left(\frac{1}{2}\right)\right) \\
& \stackrel{\mu_{-}^{\bullet}}{\longleftrightarrow}(\boldsymbol{r}(B), x(1), y(1)) \stackrel{\mu_{+}^{\bullet} \mu_{-}^{\circ}}{\longleftrightarrow}\left(-\boldsymbol{r}(B), x\left(\frac{3}{2}\right), y\left(\frac{3}{2}\right)\right) \stackrel{\mu_{-}^{\bullet}}{\longleftrightarrow} \cdots,
\end{aligned}
$$

where $\boldsymbol{r}(B)=B^{\prime}$ is defined by $B_{\boldsymbol{r}(\mathbf{i}) \boldsymbol{r}(\mathbf{j})}^{\prime}=B_{\mathbf{i j}}$.

For a pair $(\mathbf{i}, u) \in \mathbf{I} \times \frac{1}{2} \mathbb{Z}$, we define the parity conditions $\mathbf{p}_{+}$and $\mathbf{p}_{-}$by

$$
\mathbf{p}_{+}:\left\{\begin{array}{ll}
\mathbf{i} \in \mathbf{I}_{+}^{\circ} \sqcup \mathbf{I}_{+}^{\bullet}, & u \equiv 0, \\
\mathbf{i} \in \mathbf{I}_{-}^{\bullet}, & u \equiv \frac{1}{2}, \frac{3}{2}, \\
\mathbf{i} \in \mathbf{I}_{-}^{\circ} \sqcup \mathbf{I}_{+}^{\bullet}, & u \equiv 1,
\end{array} \quad \mathbf{p}_{-}: \begin{cases}\mathbf{i} \in \mathbf{I}_{+}^{\circ} \sqcup \mathbf{I}_{+}^{\bullet}, & u \equiv \frac{1}{2}, \\
\mathbf{i} \in \mathbf{I}_{-}^{\bullet}, & u \equiv 0,1, \\
\mathbf{i} \in \mathbf{I}_{-}^{\circ} \sqcup \mathbf{I}_{+}^{\bullet}, & u \equiv \frac{3}{2},\end{cases}\right.
$$

where $\equiv$ is equivalence modulo $2 \mathbb{Z}$. We have

$$
(\mathbf{i}, u): \mathbf{p}_{+} \Leftrightarrow\left(\mathbf{i}, u+\frac{1}{2}\right): \mathbf{p}_{-} .
$$


R. INOUe ET AL.

Each $(\mathbf{i}, u): \mathbf{p}_{+}$is a mutation point of (2.14) in the forward direction of $u$, and each $(\mathbf{i}, u): \mathbf{p}_{-}$is one in the backward direction of $u$. Notice that there are also some (i, $u$ ) which satisfy neither $\mathbf{p}_{+}$nor $\mathbf{p}_{-}$, and are not mutation points of (2.14); explicitly, they are $(\mathbf{i}, u)$ with $\mathbf{i} \in \mathbf{I}_{+}^{\circ}, u \equiv 1, \frac{3}{2} \bmod 2 \mathbb{Z}$, or with $\mathbf{i} \in \mathbf{I}_{-}^{\circ}, u \equiv 0, \frac{1}{2}$ $\bmod 2 \mathbb{Z}$. Consequently, we have the following relations for $(\mathbf{i}, u): \mathbf{p}_{ \pm}$:

$$
\begin{aligned}
& x_{\mathbf{i}}(u)=x_{\mathbf{i}}\left(u \mp \frac{1}{2}\right) \quad\left(\mathbf{i} \in \mathbf{I}^{\bullet}\right), \\
& x_{\mathbf{i}}(u)=x_{\mathbf{i}}\left(u \mp \frac{1}{2}\right)=x_{\mathbf{i}}(u \mp 1)=x_{\mathbf{i}}\left(u \mp \frac{3}{2}\right) \quad\left(\mathbf{i} \in \mathbf{I}^{\circ}\right), \\
& y_{\mathbf{i}}(u)=y_{\mathbf{i}}\left(u \pm \frac{1}{2}\right)^{-1} .
\end{aligned}
$$

There is a correspondence between the parity condition $\mathbf{p}_{+}$here and $\mathbf{P}_{+}, \mathbf{P}_{+}^{\prime}$ in $(2.5)$ and (2.8).

Lemma 2.4. Below $\equiv$ means equivalence modulo $2 \mathbb{Z}$.

(i) The map $g: \mathcal{I}_{\ell+} \rightarrow\left\{(\mathbf{i}, u): \mathbf{p}_{+}\right\}$given by

$$
\left(a, m, u-\frac{1}{t_{a}}\right) \mapsto \begin{cases}((a, m), u), & a \neq r ; r+a+m+u \equiv 1, \\ ((2 r-a, m), u), & a \neq r ; r+a+m+u \equiv 0, \\ ((r, m), u), & a=r,\end{cases}
$$

is a bijection.

(ii) The map $g^{\prime}: \mathcal{I}_{\ell+}^{\prime} \rightarrow\left\{(\mathbf{i}, u): \mathbf{p}_{+}\right\}$given by

$$
(a, m, u) \mapsto \begin{cases}((a, m), u), & a \neq r ; r+a+m+u \equiv 1, \\ ((2 r-a, m), u), & a \neq r ; r+a+m+u \equiv 0, \\ ((r, m), u), & a=r,\end{cases}
$$

is a bijection.

Proof. Look at the example in Figures $2-5$.

We introduce alternative labels $x_{\mathbf{i}}(u)=x_{m}^{(a)}\left(u-1 / t_{a}\right)\left(\left(a, m, u-1 / t_{a}\right) \in \mathcal{I}_{\ell+}\right)$ for $(\mathbf{i}, u)=g\left(\left(a, m, u-1 / t_{a}\right)\right)$ and $y_{\mathbf{i}}(u)=y_{m}^{(a)}(u)\left((a, m, u) \in \mathcal{I}_{\ell+}^{\prime}\right)$ for $(\mathbf{i}, u)=$ $g^{\prime}((a, m, u))$, respectively, as in Figures 2-5. They will be used below (and also in Section 6) to relate the T-systems and Y-systems with $\mathcal{A}(B, x, y)$ and $\mathcal{G}(B, y)$.

\section{$\S 2.5$. T-system and cluster algebra}

The T-system $\mathbb{T}_{\ell}\left(B_{r}\right)$ naturally appears as a system of relations among the cluster variables $x_{\mathbf{i}}(u)$ in the trivial evaluation of coefficients. (The quiver $Q_{\ell}\left(B_{r}\right)$ is designed to do so.) Let $\mathcal{A}(B, x)$ be the cluster algebra with trivial coefficients, where 


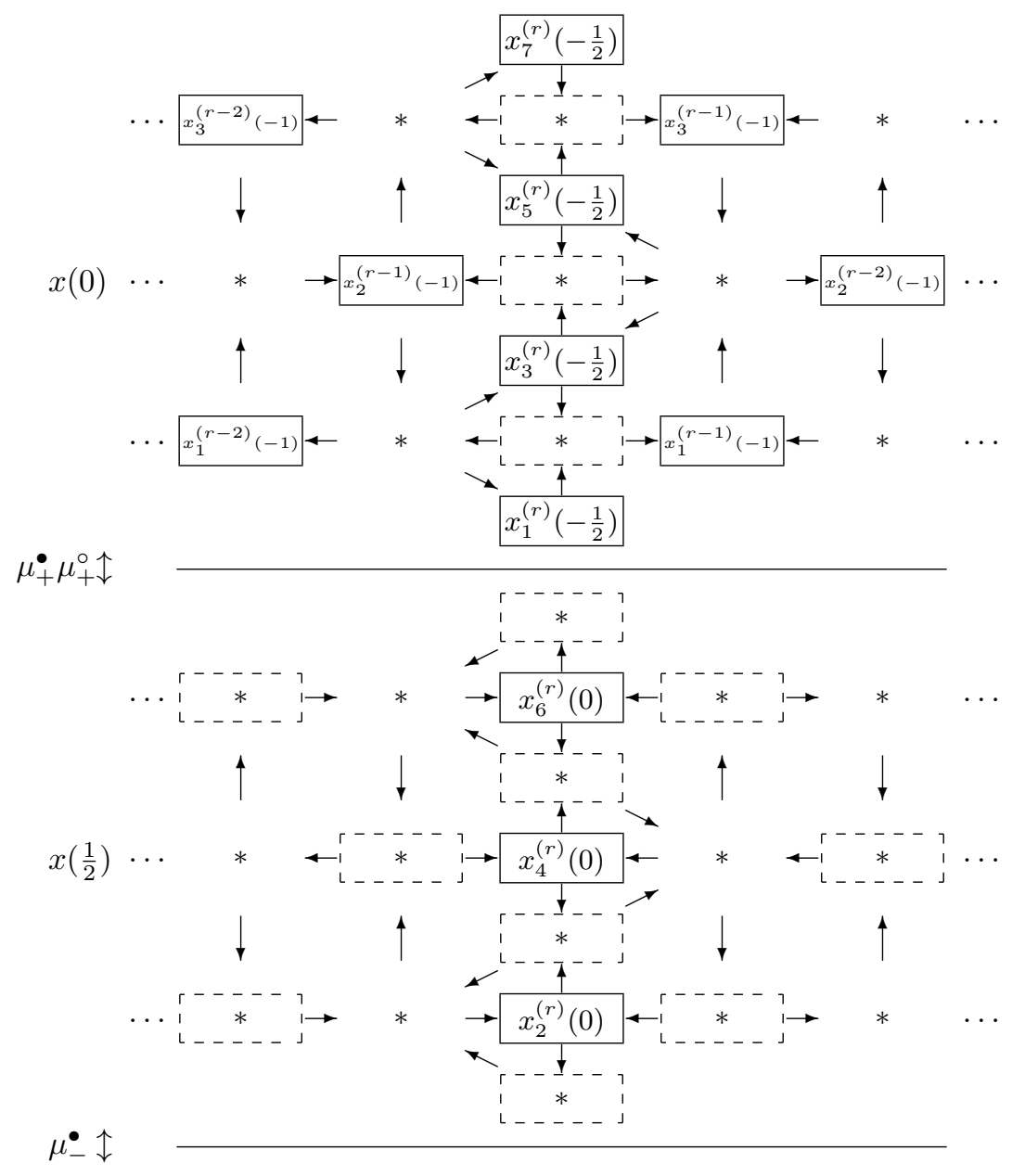

Figure 2. (Continued in Figure 3.) Labeling of the cluster variables $x_{\mathbf{i}}(u)$ by $\mathcal{I}_{\ell+}$ for $B_{r}, \ell=4$. The variables framed by solid/dashed lines satisfy the condition $\mathbf{p}_{+} / \mathbf{p}_{-}$, respectively.

$(B, x)$ is the initial seed. Let $\mathbf{1}=\{1\}$ be the trivial semifield and $\pi_{\mathbf{1}}: \mathbb{Q}_{\mathrm{sf}}(y) \rightarrow \mathbf{1}$, $y_{\mathbf{i}} \mapsto 1$, be the projection. Let $\left[x_{\mathbf{i}}(u)\right]_{1}$ denote the image of $x_{\mathbf{i}}(u)$ under the algebra homomorphism $\mathcal{A}(B, x, y) \rightarrow \mathcal{A}(B, x)$ induced by $\pi_{\mathbf{1}}$. It is called the trivial evaluation.

Recall that $G(b, k, v ; a, m, u)$ is defined in (1.5). 


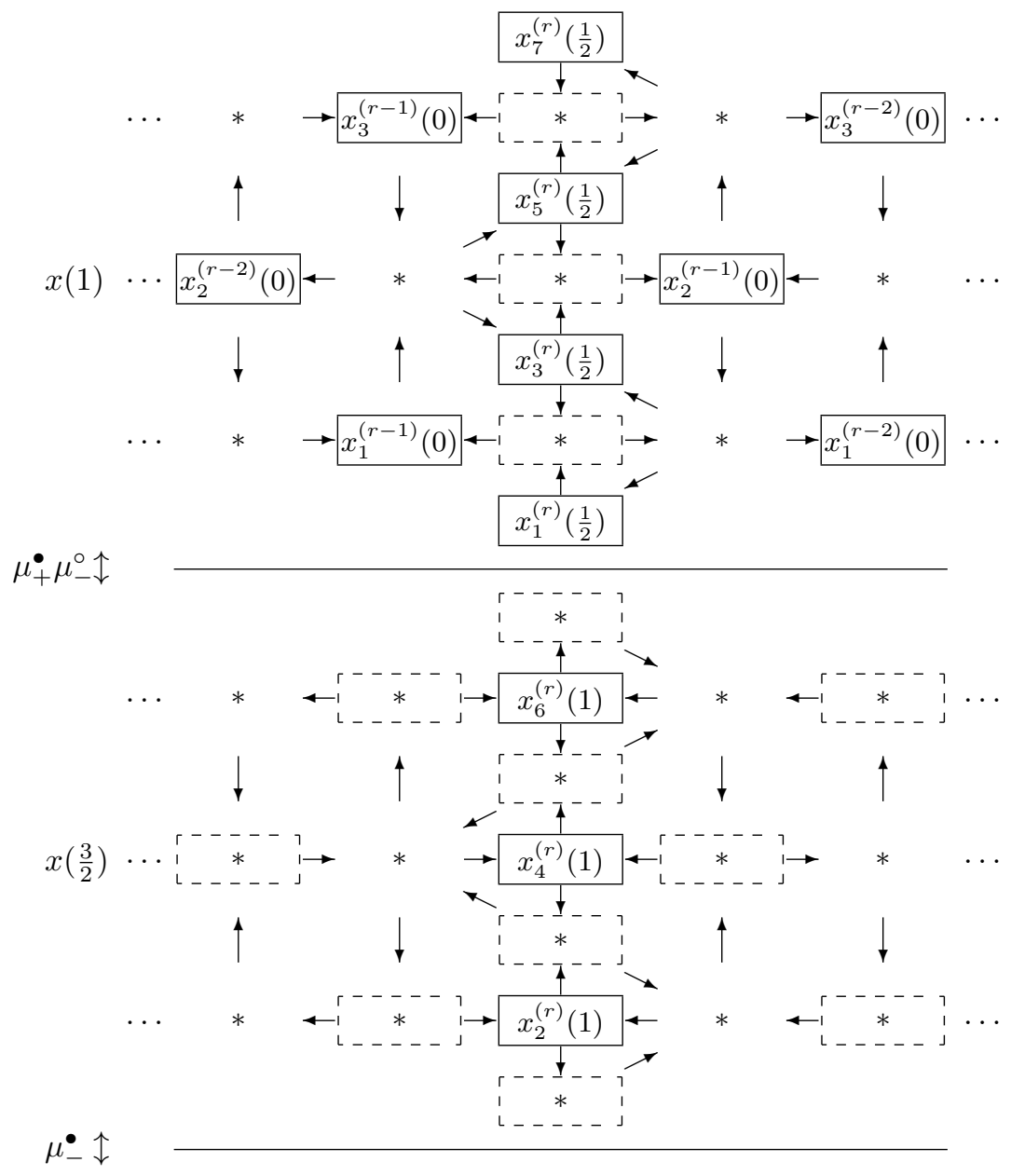

Figure 3. Continuation of Figure 2.

Lemma 2.5. The family $\left\{x_{m}^{(a)}(u) \mid(a, m, u) \in \mathcal{I}_{\ell+}\right\}$ satisfies the system of relations

$(2.22) \quad x_{m}^{(a)}\left(u-\frac{1}{t_{a}}\right) x_{m}^{(a)}\left(u+\frac{1}{t_{a}}\right)=\frac{y_{m}^{(a)}(u)}{1+y_{m}^{(a)}(u)} \prod_{(b, k, v) \in \mathcal{I}_{\ell+}} x_{k}^{(b)}(v)^{G(b, k, v ; a, m, u)}$

$$
+\frac{1}{1+y_{m}^{(a)}(u)} x_{m-1}^{(a)}(u) x_{m+1}^{(a)}(u),
$$

where $(a, m, u) \in \mathcal{I}_{+\ell}^{\prime}$. In particular, the family $\left\{\left[x_{m}^{(a)}(u)\right]_{\mathbf{1}} \mid(a, m, u) \in \mathcal{I}_{\ell+}\right\}$ satisfies the T-system $\mathbb{T}_{\ell}\left(B_{r}\right)$ in $\mathcal{A}(B, x)$ after replacing $T_{m}^{(a)}(u)$ with $\left[x_{m}^{(a)}(u)\right]_{1}$. 


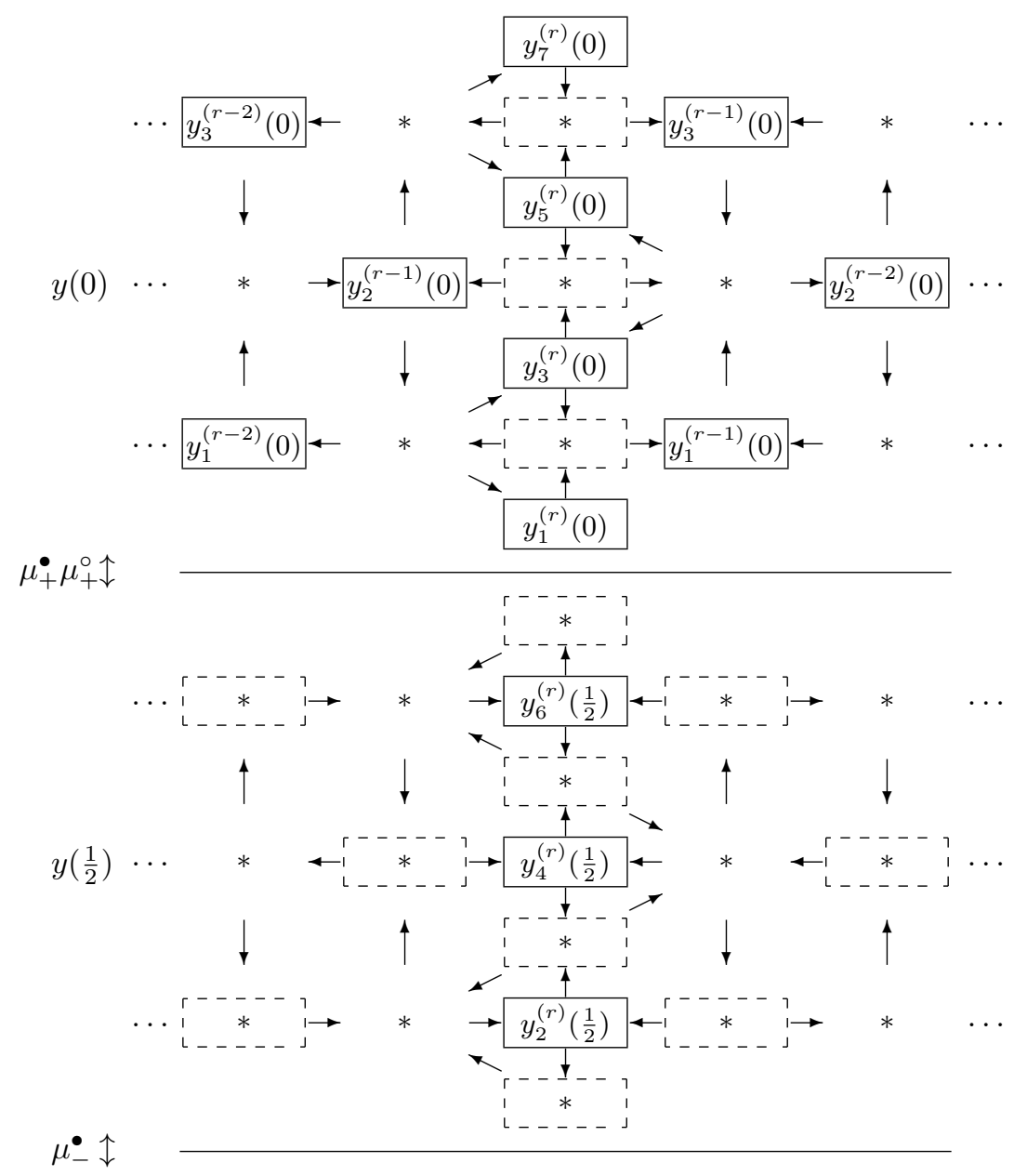

Figure 4. Continuation of Figure 5. Labeling of the coefficients $y_{\mathbf{i}}(u)$ by $\mathcal{I}_{\ell+}^{\prime}$ for $B_{r}$, $\ell=4$. The variables framed by solid/dashed lines satisfy the condition $\mathbf{p}_{+} / \mathbf{p}_{-}$, respectively.

Proof. An easy way to prove this is to represent all the relevant cluster variables in Figures 2 and 3 by $x_{m}^{(a)}(u)(a, m, u) \in \mathcal{I}_{\ell+}$, and then to apply mutations at $(\mathbf{i}, u): \mathbf{p}_{+}$in the figures. For example, consider the mutation at $((r, 3), 0): \mathbf{p}_{+}$. Then, by the exchange relation, $x_{3}^{(r)}\left(-\frac{1}{2}\right)$ is mutated to

$$
\frac{1}{x_{3}^{(r)}\left(-\frac{1}{2}\right)}\left\{\frac{y_{3}^{(r)}(0)}{1+y_{3}^{(r)}(0)} x_{1}^{(r-1)}(0) x_{2}^{(r-1)}(0)+\frac{1}{1+y_{3}^{(r)}(0)} x_{2}^{(r)}(0) x_{4}^{(r)}(0)\right\},
$$




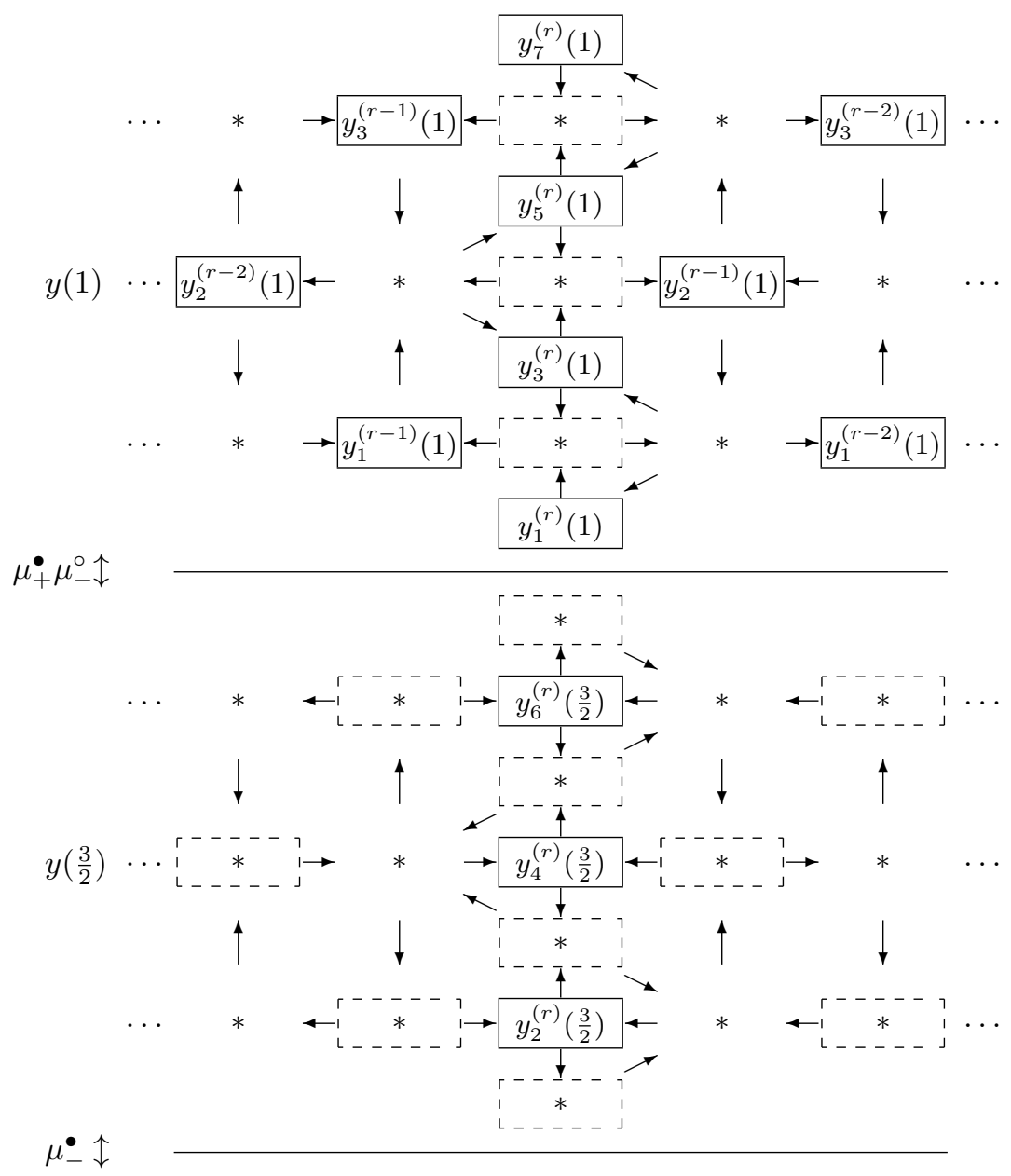

Figure 5. Continuation of Figure 4.

which should be equal to $x_{3}^{(r)}\left(\frac{1}{2}\right)$. We note that Figures 2 and 3 are general enough for that purpose.

Definition 2.6. The T-subalgebra $\mathcal{A}_{T}(B, x)$ of $\mathcal{A}(B, x)$ associated with the sequence (2.14) is the subalgebra of $\mathcal{A}(B, x)$ generated by $\left[x_{\mathbf{i}}(u)\right]_{\mathbf{1}}\left((\mathbf{i}, u) \in \mathbf{I} \times \frac{1}{2} \mathbb{Z}\right)$.

Theorem 2.7. The ring $\mathcal{T}_{\ell}^{\circ}\left(B_{r}\right)_{+}$is isomorphic to $\mathcal{A}_{T}(B, x)$ via the correspondence $T_{m}^{(a)}(u) \mapsto\left[x_{m}^{(a)}(u)\right]_{\mathbf{1}}$. 
Proof. First we note that $\mathcal{A}_{T}(B, x)$ is generated by $\left[x_{\mathbf{i}}(u)\right]_{\mathbf{1}}\left((\mathbf{i}, u): \mathbf{p}_{+}\right)$by $(2.17)$ and (2.18). Then the claim follows from Lemma 2.5 in the same way as [IIKNS, Proposition 4.24].

\section{§2.6. Y-system and cluster algebra}

The Y-system $\mathbb{Y}_{\ell}\left(B_{r}\right)$ also naturally appears as a system of relations among the coefficients $y_{\mathbf{i}}(u)$.

Lemma 2.8. The family $\left\{y_{m}^{(a)}(u) \mid(a, m, u) \in \mathcal{I}_{\ell+}^{\prime}\right\}$ satisfies the $Y$-system $\mathbb{Y}_{\ell}\left(B_{r}\right)$ after replacing $Y_{m}^{(a)}(u)$ with $y_{m}^{(a)}(u)$.

Proof. This can be easily shown using Figures 4 and 5. For example, consider the mutation at $((r-1,2), 0): \mathbf{p}_{+}$. The coefficient $y_{2}^{(r-1)}(0)$ is mutated to $y_{2}^{(r-1)}(0)^{-1}$. Then, at $u=\frac{1}{2}, 1, \frac{3}{2}, y_{2}^{(r-1)}(0)^{-1}$ is multiplied by the factors

$$
\begin{array}{lll}
1+y_{4}^{(r)}\left(\frac{1}{2}\right), & \frac{y_{3}^{(r-1)}(1)}{1+y_{1}^{(r-1)}(1)}, & \frac{y_{3}^{(r-1)}(1)}{1+y_{1}^{(r-1)}(1)} \\
1+y_{3}^{(r)}(1), & 1+y_{5}^{(r)}(1), & 1+y_{4}^{(r)}\left(\frac{3}{2}\right) .
\end{array}
$$

The result should be equal to $y_{2}^{(r-1)}(2)$.

Definition 2.9. The $Y$-subgroup $\mathcal{G}_{Y}(B, y)$ of $\mathcal{G}(B, y)$ associated with the sequence (2.14) is the subgroup of $\mathcal{G}(B, y)$ generated by $y_{\mathbf{i}}(u)\left((\mathbf{i}, u) \in \mathbf{I} \times \frac{1}{2} \mathbb{Z}\right)$ and $1+y_{\mathbf{i}}(u)$ $\left((\mathbf{i}, u): \mathbf{p}_{+}\right.$or $\left.\mathbf{p}_{-}\right)$.

Notice that we excluded $1+y_{\mathbf{i}}(u)$ for $(\mathbf{i}, u)$ satisfying neither $\mathbf{p}_{+}$nor $\mathbf{p}_{-}$. This is because such an $(\mathbf{i}, u)$ is not a mutation point so that the factor $1+y_{\mathbf{i}}(u)$ does not appear anywhere for the mutation sequence (2.14).

Theorem 2.10. The group $\mathrm{y}_{\ell}^{\circ}\left(B_{r}\right)_{+}$is isomorphic to $\mathcal{G}_{Y}(B, y)$ via the correspondence $Y_{m}^{(a)}(u) \mapsto y_{m}^{(a)}(u)$ and $1+Y_{m}^{(a)}(u) \mapsto 1+y_{m}^{(a)}(u)$.

Proof. We note that $\mathcal{G}_{Y}(B, y)$ is generated by $y_{\mathbf{i}}(u), 1+y_{\mathbf{i}}(u)\left((\mathbf{i}, u): \mathbf{p}_{+}\right)$by $(2.19)$. Then the claim follows from Lemma 2.8 in the same way as [KNS3, Theorem $6.19]$.

\section{§3. Tropical Y-system at level 2}

In this section we study the tropical version of the Y-system at level 2. 


\section{§3.1. Tropical Y-system}

Let $y=y(0)$ be the initial coefficient tuple of the cluster algebra $\mathcal{A}(B, x, y)$ with $B=B_{\ell}\left(B_{r}\right)$ of the previous section. Let Trop $(y)$ be the tropical semifield for $y$. Let $\pi_{\mathbf{T}}: \mathbb{Q}_{\mathrm{sf}}(y) \rightarrow \operatorname{Trop}(y), y_{\mathbf{i}} \mapsto y_{\mathbf{i}}$, be the projection. Let $\left[y_{\mathbf{i}}(u)\right]_{\mathbf{T}}$ and $\left[\mathcal{G}_{Y}(B, y)\right]_{\mathbf{T}}$ denote the images of $y_{\mathbf{i}}(u)$ and $\mathcal{G}_{Y}(B, y)$ under the multiplicative group homomorphism induced from $\pi_{\mathbf{T}}$, respectively. They are called the tropical evaluations, and the resulting relations in the group $\left[\mathcal{G}_{Y}(B, y)\right]_{\mathbf{T}}$ form the tropical $Y$-system. They we are first studied in [FZ2] for simply laced type at level 2 in our terminology.

We say a (Laurent) monomial $m=\prod_{\mathbf{i} \in \mathbf{I}} y_{\mathbf{i}}^{k_{\mathbf{i}}}$ is positive (resp. negative) if $m \neq 1$ and $k_{\mathbf{i}} \geq 0$ (resp. $\left.k_{\mathbf{i}} \leq 0\right)$ for any $\mathbf{i}$.

The next 'tropical mutation rule' for $\left[y_{\mathbf{i}}(u)\right]_{\mathbf{T}}$ is general and useful.

Lemma 3.1. Suppose that $y^{\prime \prime}$ is the coefficient tuple obtained from the mutation of another coefficient tuple $y^{\prime}$ at $\mathbf{k}$ with mutation matrix $B^{\prime}$. Then, for any $\mathbf{i} \neq \mathbf{k}$, we have the rule:

(i) $\left[y_{\mathbf{i}}^{\prime \prime}\right]_{\mathbf{T}}=\left[y_{\mathbf{i}}^{\prime}\right]_{\mathbf{T}}\left[y_{\mathbf{k}}^{\prime}\right]_{\mathbf{T}}$ if one of the following conditions holds:

(a) $B_{\mathbf{k i}}^{\prime}>0$, and $\left[y_{\mathbf{k}}^{\prime}\right]_{\mathbf{T}}$ is positive.

(b) $B_{\mathbf{k i}}^{\prime}<0$, and $\left[y_{\mathbf{k}}^{\prime}\right]_{\mathbf{T}}$ is negative.

(ii) $\left[y_{\mathbf{i}}^{\prime \prime}\right]_{\mathbf{T}}=\left[y_{\mathbf{i}}^{\prime}\right]_{\mathbf{T}}$ if one of the following conditions holds:
(a) $B_{\mathbf{k i}}^{\prime}=0$.
(b) $B_{\mathbf{k i}}^{\prime}>0$, and $\left[y_{\mathbf{k}}^{\prime}\right]_{\mathbf{T}}$ is negative.
(c) $B_{\mathbf{k i}}^{\prime}<0$, and $\left[y_{\mathbf{k}}^{\prime}\right]_{\mathbf{T}}$ is positive.

Proof. An immediate consequence of the exchange relation (2.3) and (2.1).

The following properties of the tropical Y-system at level 2 will be the key in the entire method.

Proposition 3.2. For $\left[\mathcal{G}_{Y}(B, y)\right]_{\mathbf{T}}$ with $B=B_{2}\left(B_{r}\right)$, the following facts hold:

(i) Let $0 \leq u<2$. For any $(\mathbf{i}, u): \mathbf{p}_{+}$, the monomial $\left[y_{\mathbf{i}}(u)\right]_{\mathbf{T}}$ is positive.

(ii) Let $-h^{\vee} \leq u<0$.

(a) Let $\mathbf{i} \in \mathbf{I}^{\circ} \sqcup \mathbf{I}_{-}^{\bullet}$. For any $(\mathbf{i}, u): \mathbf{p}_{+}$, the monomial $\left[y_{\mathbf{i}}(u)\right]_{\mathbf{T}}$ is negative.

(b) Let $\mathbf{i} \in \mathbf{I}_{+}^{\mathbf{0}}$. For any $(\mathbf{i}, u): \mathbf{p}_{+}$, the monomial $\left[y_{\mathbf{i}}(u)\right]_{\mathbf{T}}$ is negative for $u=-1,-3, \ldots$ and positive for $u=-2,-4, \ldots$

(iii) $y_{i i^{\prime}}(2)=y_{i i^{\prime}}^{-1}$ if $i \neq r$ and $y_{r, 4-i^{\prime}}^{-1}$ if $i=r$.

(iv) $y_{i i^{\prime}}\left(-h^{\vee}\right)=y_{2 r-i, i^{\prime}}^{-1}$. 
Example 3.3. Consider the simplest case $B_{2}$. All the coefficients $\left[y_{\mathbf{i}}(u)\right]_{\mathbf{T}}$ in the region $-3 \leq u \leq 2$ are calculated from Lemma 3.1 and explicitly given in Figure 6 . We continue to use the convention in Figures $2-5$ that the variables framed by solid/dashed lines satisfy the condition $\mathbf{p}_{+} / \mathbf{p}_{-}$. We recall that they are the mutation points for the forward/backward direction of $u$. In Figure 6, the configuration

$$
11
$$

for example represents the monomial $y_{11} y_{22}$, where $\cdot$ stands for 0 . One can observe all the properties of Proposition 3.2 in Figure 6. Let us further observe that, in the region $-3 \leq u<0$, we have six negative monomials for $\mathbf{i} \in \mathbf{I}^{\circ} \sqcup \mathbf{I}_{-}^{\bullet},(\mathbf{i}, u): \mathbf{p}_{+}$,

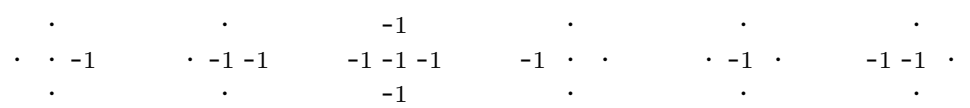

If we concentrate on the middle row, they naturally correspond to the positive roots of type $A_{3}$,

$$
\alpha_{1}, \alpha_{2}, \alpha_{3}, \alpha_{1}+\alpha_{2}, \alpha_{2}+\alpha_{3}, \alpha_{1}+\alpha_{2}+\alpha_{3}
$$

\section{§3.2. Proof of Proposition 3.2}

For general $r$, one can directly verify (i) and (iii) as in Figure 6.

Note that (ii) and (iv) can be proved independently for each variable $y_{\mathbf{i}}$. (To be precise, we also need to ensure that no monomial is 1 . However, this can be easily guaranteed, so that we do not give the details here.) As for the powers of $y_{r 1}$ and $y_{r 3}$, it is easy to verify the claim by direct calculations. Therefore, it is enough to prove (ii) and (iv) only for the powers of variables $y_{i 1}(i \neq r)$ and $y_{r 2}$. To do that, we use the description of the tropical Y-system in the region $-h^{\vee} \leq u<0$ by the root system of type $A_{2 r-1}$, in the spirit of [FZ2].

Let $A_{2 r-1}$ be the Dynkin diagram of type $A$ with index set $J=\{1, \ldots, 2 r-1\}$. We assign a sign $+/-$ to each vertex of $A_{2 r-1}$ (except for $r$ ) of $A_{2 r-1}$ as inherited from $Q_{2}\left(B_{r}\right)$ :

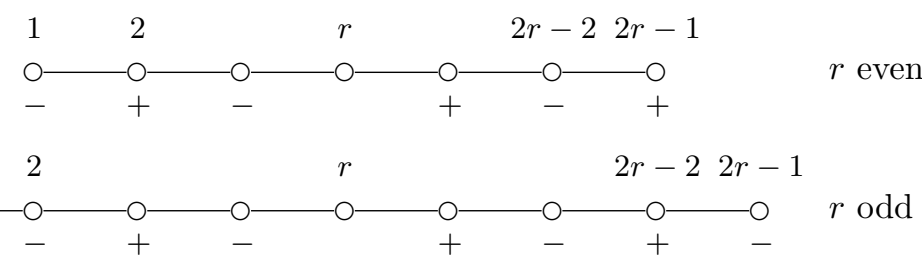




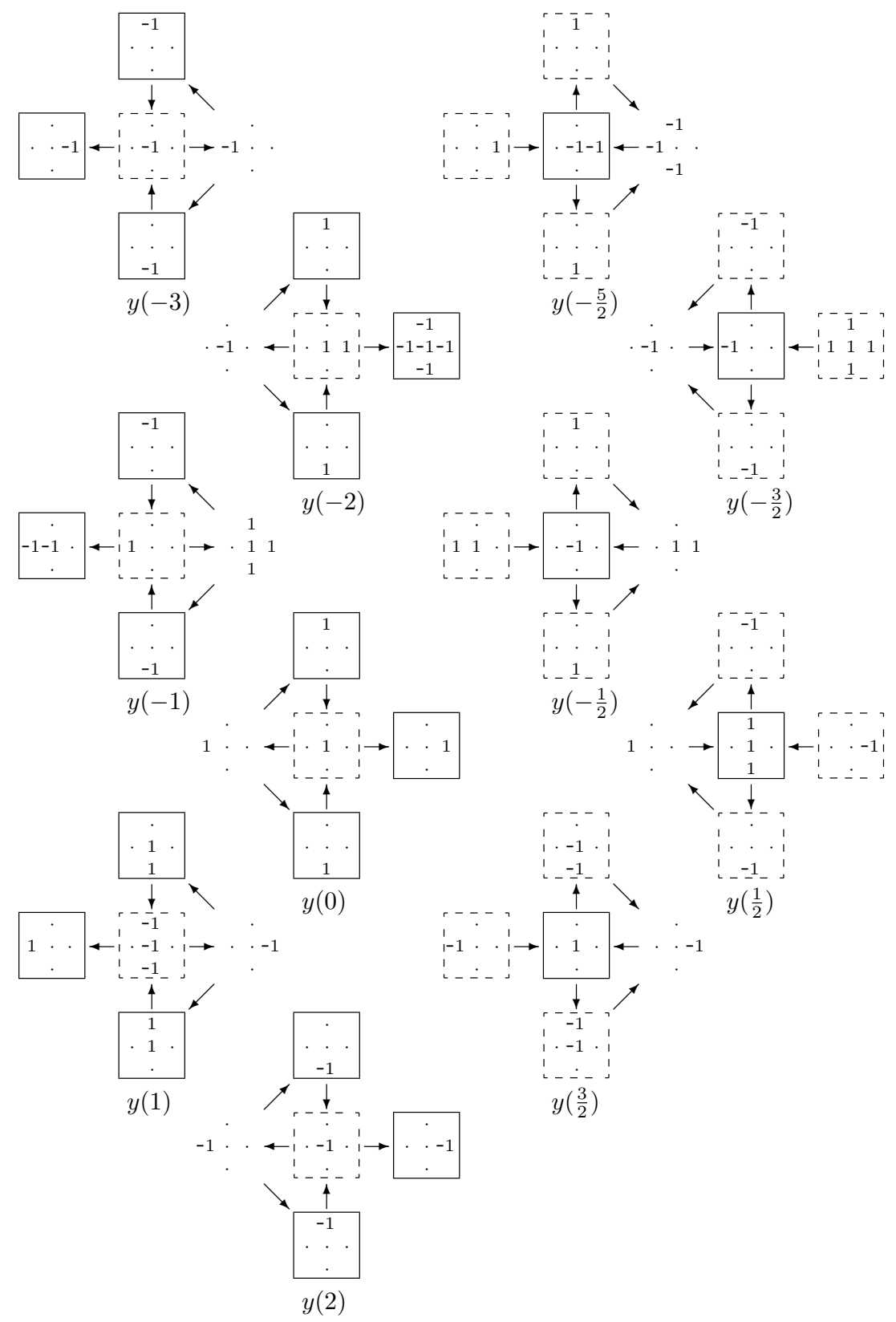

Figure 6. Tropical Y-system of type $B_{2}$ at level 2 . We continue to use the convention in Figures $2-5$ that the variables framed by solid/dashed lines satisfy the condition $\mathbf{p}_{+} / \mathbf{p}_{-}$. 
Let $\Pi=\left\{\alpha_{1}, \ldots, \alpha_{2 r-1}\right\},-\Pi$ and $\Phi_{+}$be the set of simple roots, negative simple roots and positive roots, respectively, of type $A_{2 r-1}$. Following [FZ2], we introduce the piecewise-linear analogue $\sigma_{i}$ of the simple reflection $s_{i}$, acting on the set $\Phi_{\geq-1}=\Phi_{+} \sqcup(-\Pi)$ of almost positive roots, by

$$
\begin{gathered}
\sigma_{i}(\alpha)=s_{i}(\alpha), \quad \alpha \in \Phi_{+}, \\
\sigma_{i}\left(-\alpha_{j}\right)= \begin{cases}\alpha_{j}, & j=i, \\
-\alpha_{j}, & \text { otherwise. }\end{cases}
\end{gathered}
$$

Let

$$
\sigma_{+}=\prod_{i \in J_{+}} \sigma_{i}, \quad \sigma_{-}=\prod_{i \in J_{-}} \sigma_{i}
$$

where $J_{ \pm}$is the set of vertices of $A_{2 r-1}$ with property \pm . We define

$$
\sigma=\sigma_{r} \sigma_{-} \sigma_{r} \sigma_{+}
$$

Let $\omega: i \rightarrow 2 r-i$ be an involution on $J$.

Lemma 3.4. The following facts hold:

(i) For $i \in J_{+}, \sigma^{k}\left(-\alpha_{i}\right) \in \Phi_{+}(1 \leq k \leq r-1), \sigma^{r}\left(-\alpha_{i}\right)=-\alpha_{\omega(i)}$.

(ii) For $i \in J_{-}, \sigma^{k}\left(-\alpha_{i}\right) \in \Phi_{+}(1 \leq k \leq r), \sigma^{r+1}\left(-\alpha_{i}\right)=-\alpha_{\omega(i)}$.

(iii) $\sigma^{k}\left(-\alpha_{r}\right) \in \Phi_{+}(1 \leq k \leq r-1), \sigma^{r}\left(-\alpha_{r}\right)=-\alpha_{r}$.

(iv) $\sigma^{k}\left(\alpha_{r}\right) \in \Phi_{+}(0 \leq k \leq r-1), \sigma^{r}\left(\alpha_{r}\right)=\alpha_{r}$.

(v) The elements in $\Phi_{+}$in (i)-(iv) exhaust the set $\Phi_{+}$, thereby providing the orbit decomposition of $\Phi_{+}$by $\sigma$.

Proof. (i)-(iv). These are verified by explicitly calculating $\sigma^{k}\left(-\alpha_{i}\right)(i \in J)$ and $\sigma^{k}\left(\alpha_{r}\right)$. The example for $r=6$ is given in Table 1 , where we use the notation

$$
[i, j]=\alpha_{i}+\cdots+\alpha_{j} \quad(i<j), \quad[i]=\alpha_{i}
$$

In fact, it is not difficult to read off the general rule from this example.

(v) The total number of elements in $\Phi_{+}$in (i)-(iv) is $r(2 r-1)$, which coincides with $\left|\Phi_{+}\right|$.

The orbit of $\sigma\left(-\alpha_{i}\right)(i \neq r)$ is further described by the root system of type $A_{2 r-2}$. 
R. InOUe et AL.

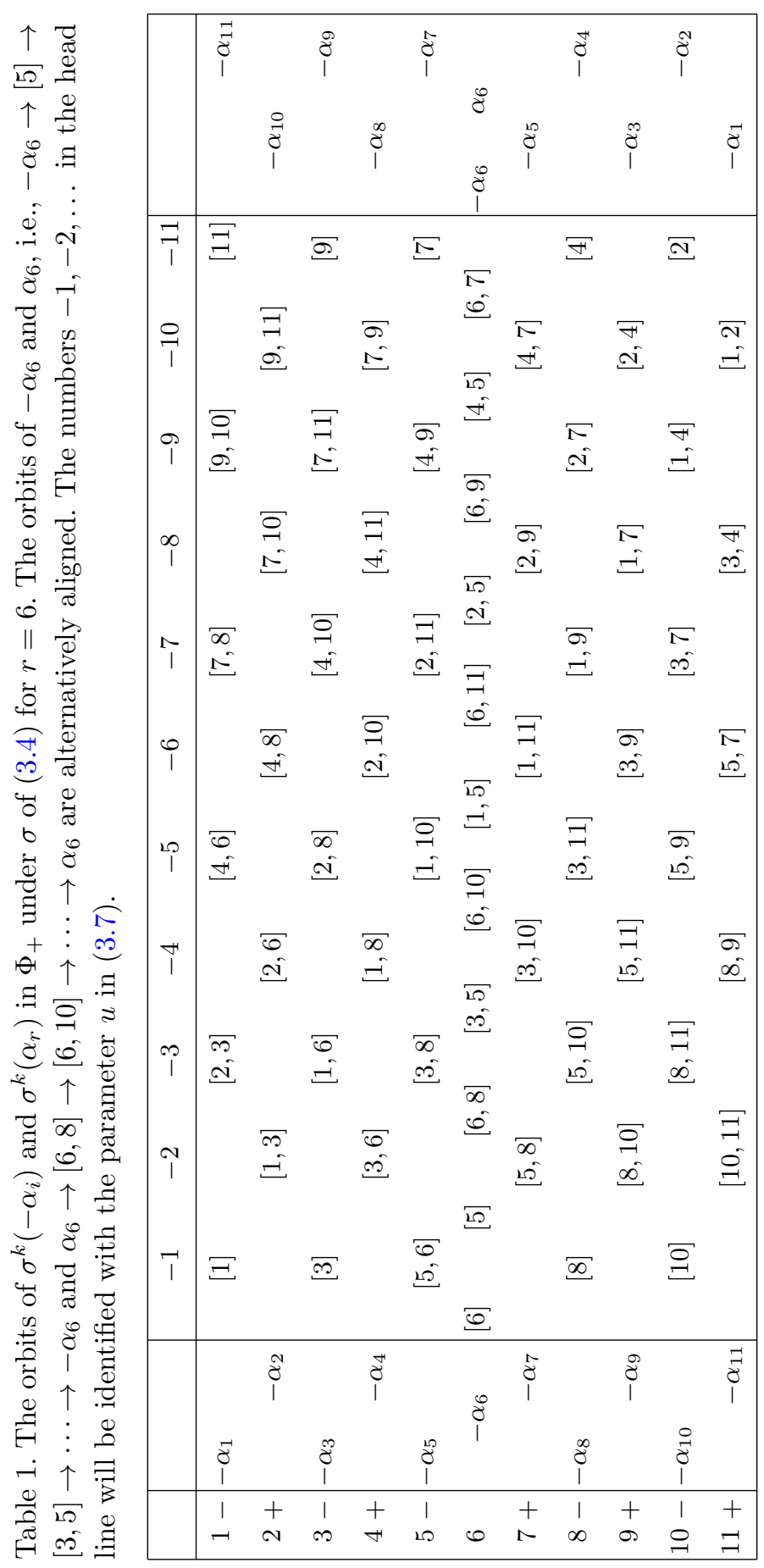


Lemma 3.5. Let $O_{i}=\left\{\sigma^{k}\left(-\alpha_{i}\right) \mid 1 \leq k \leq r-1\right\}$ for $i \in J_{+}$and $O_{i}=\left\{\sigma^{k}\left(-\alpha_{i}\right) \mid\right.$ $1 \leq k \leq r\}$ for $i \in J_{-}$. Let $\Phi_{+}^{\prime}$ be the set of positive roots of type $A_{2 r-2}$ with index set $J^{\prime}=J-\{r\}$, and

$$
\rho: \bigsqcup_{i \in J^{\prime}} O_{i} \rightarrow \Phi_{+}^{\prime}
$$

be the map which removes $\alpha_{r}$ from $\alpha$ if $\alpha$ contains $\alpha_{r}$, and does nothing otherwise. Then $\rho$ is a bijection, and its inverse $\rho^{-1}\left(\alpha^{\prime}\right)$ adds $\alpha_{r}$ if $\alpha^{\prime}$ contains $\alpha_{r-1}$, and does nothing otherwise. Furthermore, under the bijection $\rho$, the action of $\sigma$ is translated into the Coxeter element $s=s_{-} s_{+}$of type $A_{2 r-2}$ acting on $\Phi_{+}^{\prime}$, where $s_{ \pm}=\prod_{i \in J_{ \pm}} s_{i}$.

$$
\text { For }-h^{\vee} \leq u<0 \text {, define }
$$

$$
\alpha_{i}(u)= \begin{cases}\sigma^{-u / 2}\left(-\alpha_{i}\right), & i \in J_{+}, u \equiv 0, \\ \sigma^{-(u-1) / 2}\left(-\alpha_{i}\right), & i \in J_{-}, u \equiv-1, \\ \sigma^{-(2 u-1) / 4}\left(-\alpha_{r}\right), & i=r, u \equiv-\frac{3}{2} \\ \sigma^{-(2 u+1) / 4}\left(\alpha_{r}\right), & i=r, u \equiv-\frac{1}{2}\end{cases}
$$

where $\equiv$ is equivalence modulo $2 \mathbb{Z}$. Note that they correspond to the positive roots in Table 1 with $u$ being the parameter in the head line. By Lemma 3.4 these are all the positive roots of $A_{2 r-1}$.

Lemma 3.6. The family in (3.7) satisfies the recurrence relations

$$
\begin{aligned}
\alpha_{i}(u-1)+\alpha_{i}(u+1) & =\alpha_{i-1}(u)+\alpha_{i+1}(u) \quad(i \neq r-1, r, r+1), \\
\alpha_{r-1}(u-1)+\alpha_{r-1}(u+1) & =\alpha_{r-2}(u)+\alpha_{r+1}(u), \\
\alpha_{r+1}(u-1)+\alpha_{r+1}(u+1) & =\alpha_{r-1}(u)+\alpha_{r+2}(u), \\
\alpha_{r}\left(u-\frac{1}{2}\right)+\alpha_{r}\left(u+\frac{1}{2}\right) & =\alpha_{r-1}(u) \quad(u \text { odd }), \\
\alpha_{r}\left(u-\frac{1}{2}\right)+\alpha_{r}\left(u+\frac{1}{2}\right) & =\alpha_{r+1}(u) \quad(u \text { even }),
\end{aligned}
$$

where $\alpha_{0}(u)=\alpha_{2 r}(u)=0$.

Proof. These relations are easily verified by using the explicit expressions of $\alpha_{i}(u)$. See Table 1. The first three relations can also be obtained from Lemma 3.5 and [FZ3, Eq. (10.9)].

Let us return to the proof of (ii) of Proposition 3.2 for the powers of the variables $y_{i 1}(i \neq r)$ and $y_{r 2}$. For a monomial $m$ in $y=\left(y_{\mathbf{i}}\right)_{\mathbf{i} \in \mathbf{I}}$, let $\pi_{A}(m)$ denote the specialization with $y_{r 1}=y_{r 3}=1$. For simplicity, we set $y_{i 1}=y_{i}(i \neq r)$, 
$y_{r 2}=y_{r}$, and also $y_{i 1}(u)=y_{i}(u)(i \neq r), y_{r 2}(u)=y_{r}(u)$. We define the vectors $\mathbf{t}_{i}(u)=\left(t_{i}(u)_{k}\right)_{k=1}^{2 r-1}$ by

$$
\pi_{A}\left(\left[y_{i}(u)\right]_{\mathbf{T}}\right)=\prod_{k=1}^{2 r-1} y_{k}^{t_{i}(u)_{k}}
$$

We also identify each vector $\mathbf{t}_{i}(u)$ with $\alpha=\sum_{k=1}^{2 r-1} t_{i}(u)_{k} \alpha_{k} \in \mathbb{Z} \Pi$.

Proposition 3.7. Let $-h^{\vee} \leq u<0$. Then

$$
\mathbf{t}_{i}(u)=-\alpha_{i}(u)
$$

for $(i, u)$ in (3.7), and

$$
\pi_{A}\left(\left[y_{r 1}(u)\right]_{\mathbf{T}}\right)=\pi_{A}\left(\left[y_{r 3}(u)\right]_{\mathbf{T}}\right)=1, \quad u \equiv 0 \bmod 2 \mathbb{Z} .
$$

Note that these formulas determine $\pi_{A}\left(\left[y_{\mathbf{i}}(u)\right]_{\mathbf{T}}\right)$ for any $(\mathbf{i}, u): \mathbf{p}_{+}$.

Proof. We can verify the claim for $-2 \leq u \leq-\frac{1}{2}$ by direct computation. Then, by induction on $u$ in the backward direction, we can establish the claim, together with the recurrence relations among $\mathbf{t}_{i}(u)$ 's with $(i, u)$ in $(3.7)$,

$$
\begin{aligned}
\mathbf{t}_{i}(u-1)+\mathbf{t}_{i}(u+1) & =\mathbf{t}_{i-1}(u)+\mathbf{t}_{i+1}(u), \quad i \neq r-1, r, r+1, \\
\mathbf{t}_{r-1}(u-1)+\mathbf{t}_{r-1}(u+1) & =\mathbf{t}_{r-2}(u)+\mathbf{t}_{r}\left(u-\frac{1}{2}\right)+\mathbf{t}_{r}\left(u+\frac{1}{2}\right), \\
\mathbf{t}_{r+1}(u-1)+\mathbf{t}_{r+1}(u+1) & =\mathbf{t}_{r+2}(u)+\mathbf{t}_{r}\left(u-\frac{1}{2}\right)+\mathbf{t}_{r}\left(u+\frac{1}{2}\right), \\
\mathbf{t}_{r}\left(u-\frac{1}{2}\right)+\mathbf{t}_{r}\left(u+\frac{1}{2}\right) & =\mathbf{t}_{r-1}(u), \quad u \text { odd, } \\
\mathbf{t}_{r}\left(u-\frac{1}{2}\right)+\mathbf{t}_{r}\left(u+\frac{1}{2}\right) & =\mathbf{t}_{r+1}(u), \quad u \text { even. }
\end{aligned}
$$

Note that (3.12) coincides with (3.8) under (3.10). To derive (3.12), one uses mutations as in Figure 6 (or the tropical version of the Y-system $\mathbb{Y}_{2}\left(B_{r}\right)$ directly) and the positivity/negativity of $\pi_{A}\left(\left[y_{\mathbf{i}}(u)\right]_{\mathbf{T}}\right)$ resulting from (3.10) and (3.11) by induction hypothesis.

Now (ii) in Proposition 3.2 is an immediate consequence of Lemma 3.4 and Proposition 3.7. Finally, let us prove (iv), i.e., $\mathbf{t}_{i}\left(-h^{\vee}\right)=-\alpha_{\omega(i)}$. This is shown by the following formulas obtained from Lemma 3.4 and (2.19):

$$
\begin{aligned}
& \mathbf{t}_{i}\left(-h^{\vee}+1\right)=\left\{\begin{array}{ll}
-\alpha_{\omega(i)-1}-\alpha_{\omega(i)}-\alpha_{\omega(i)+1}, & i \neq r+1, \\
-\alpha_{r-2}-\alpha_{r-1}-\alpha_{r}-\alpha_{r+1}, & i=r+1,
\end{array} \quad i \in J_{+},\right. \\
& \mathbf{t}_{i}\left(-h^{\vee}+\frac{1}{2}\right)=\alpha_{\omega(i)}, \quad \mathbf{t}_{i}\left(-h^{\vee}\right)=-\alpha_{\omega(i)}, \quad i \in J_{-}, \\
& \mathbf{t}_{r}\left(-h^{\vee}+1\right)=\alpha_{r}+\alpha_{r+1}, \quad \mathbf{t}_{r}\left(-h^{\vee}+\frac{1}{2}\right)=-\alpha_{r}-\alpha_{r+1},
\end{aligned}
$$

where $\alpha_{0}=\alpha_{2 r}=0$. This completes the proof of Proposition 3.2. 


\section{§4. Tropical Y-systems at higher levels}

We have a generalization of Proposition 3.2 for the tropical Y-systems at higher levels.

Proposition 4.1. Let $\ell>2$ be an integer. For $\left[\mathcal{G}_{Y}(B, y)\right]_{\mathbf{T}}$ with $B=B_{\ell}\left(B_{r}\right)$, the following facts hold:

(i) Let $0 \leq u<\ell$. For any $(\mathbf{i}, u): \mathbf{p}_{+}$, the monomial $\left[y_{\mathbf{i}}(u)\right]_{\mathbf{T}}$ is positive.

(ii) Let $-h^{\vee} \leq u<0$.

(a) Let $\mathbf{i} \in \mathbf{I}^{\circ} \sqcup \mathbf{I}_{-}^{\bullet}$. For any $(\mathbf{i}, u): \mathbf{p}_{+}$, the monomial $\left[y_{\mathbf{i}}(u)\right]_{\mathbf{T}}$ is negative.

(b) Let $\mathbf{i} \in \mathbf{I}_{+}^{\bullet}$. For any $(\mathbf{i}, u): \mathbf{p}_{+}$, the monomial $\left[y_{\mathbf{i}}(u)\right]_{\mathbf{T}}$ is negative for $u=-1,-3, \ldots$ and positive for $u=-2,-4, \ldots$.

(iii) $y_{i i^{\prime}}(\ell)=y_{i, \ell-i^{\prime}}^{-1}$ if $i \neq r$, and $y_{r i^{\prime}}(\ell)=y_{r, 2 \ell-i^{\prime}}^{-1}$.

(iv) $y_{i i^{\prime}}\left(-h^{\vee}\right)=y_{2 r-i, i^{\prime}}^{-1}$

Proof. This is a consequence of the factorization property of the tropical Y-system found in $[\mathrm{Nkn}]$ for the simply laced case. Roughly speaking, in the region $-h^{\vee} \leq$ $u \leq 0$, the system is factorized into 'level 2 pieces', while in the region $0 \leq u \leq \ell$, it is factorized into 'type $A$ pieces'.

First, we consider the region $-h^{\vee} \leq u<0$. Let us concentrate on the simplest nontrivial example $B_{2}$ at level 3. It turns out that this example is almost general enough. In Figure 7 , all the variables $\left[y_{\mathbf{i}}(u)\right]_{\mathbf{T}}$ in the region $-2 \leq u \leq 0$ are given explicitly. It is better to view it in the backward direction from $u=0$ to -2 . If we look at only the first three rows from the bottom, we observe that the mutations occur in exactly the same pattern as in the level 2 case of Figure 6 . So is the case for the last three rows (with a $180^{\circ}$ rotation). This is the factorization property. It occurs due to the coordination of the mutation sequence (2.14) and the positivity/negativity in Proposition 3.2. For example, in Figure 7, let us look at the vertical arrows between the second and the fourth rows. At any mutation point $(\mathbf{i}, u): \mathbf{p}_{-}$these arrows are incoming while the $\left[y_{\mathbf{i}}(u)\right]_{\mathbf{T}}$ are positive by $(2.19)$ and Proposition 3.2(ii). Therefore, by Lemma 3.1, these arrows can be forgotten during mutations. For a similar reason, the variables in the third rows are not affected by mutations in the second and the fourth rows. Therefore, as long as the positivity in the second and the fourth rows continues, so does the factorization; hence (ii) holds. Moreover, (iv) holds because it does for level 2. This argument is also applicable to any rank $r$ and level $\ell$ because of the definitions of the quiver $Q_{\ell}\left(B_{r}\right)$ and the mutation sequence (2.14).

Next, we consider the region $0 \leq u<\ell$. Again, let us concentrate on the case $B_{2}$ at level 3. In Figure 8, all the variables $\left[y_{\mathbf{i}}(u)\right]_{\mathbf{T}}$ in the region $0 \leq u \leq 2$ are 
R. INOUe ET AL.

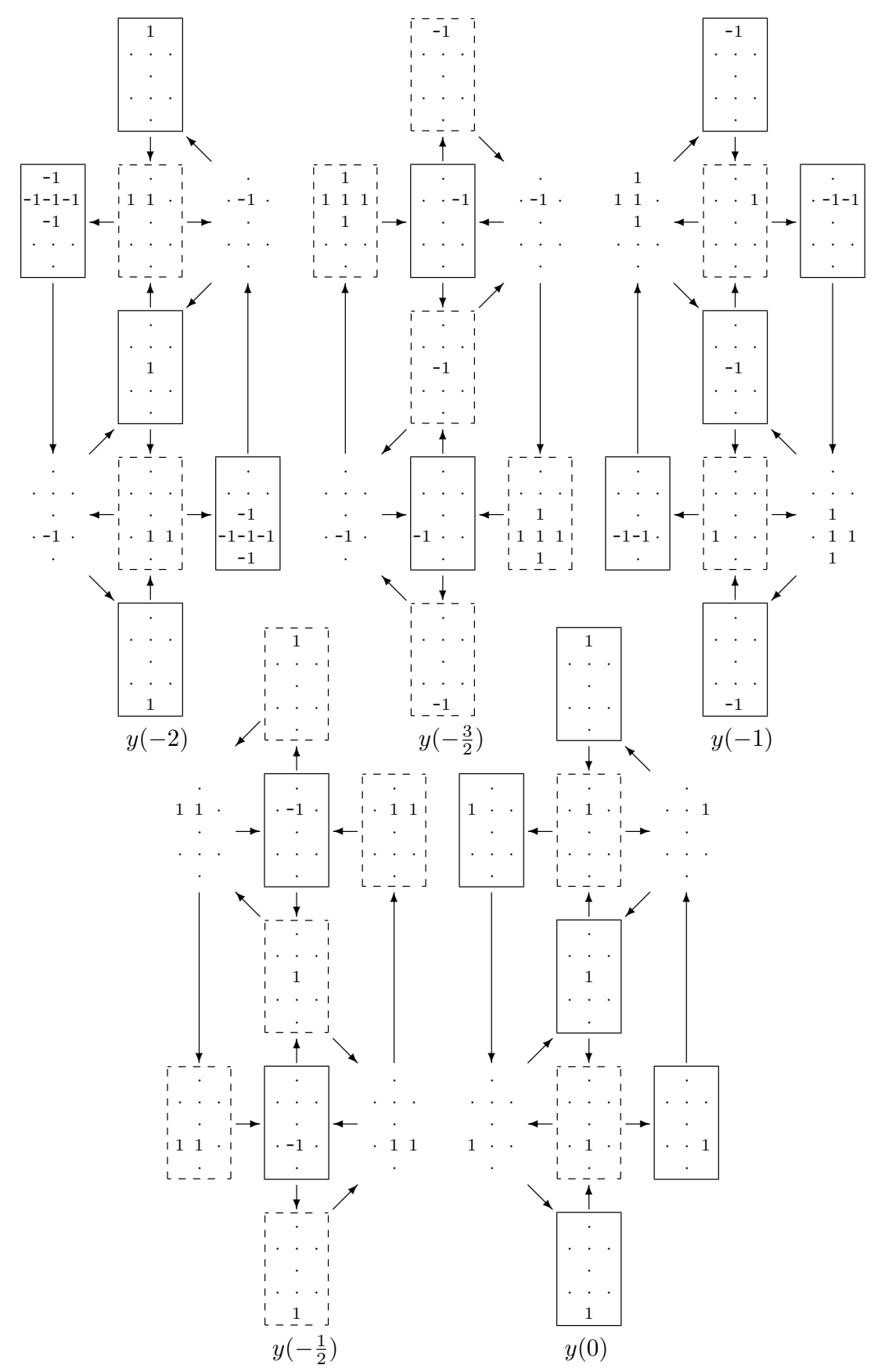

Figure 7. Tropical Y-system of type $B_{2}$ at level 3 in the region $-2 \leq u \leq 0$. 


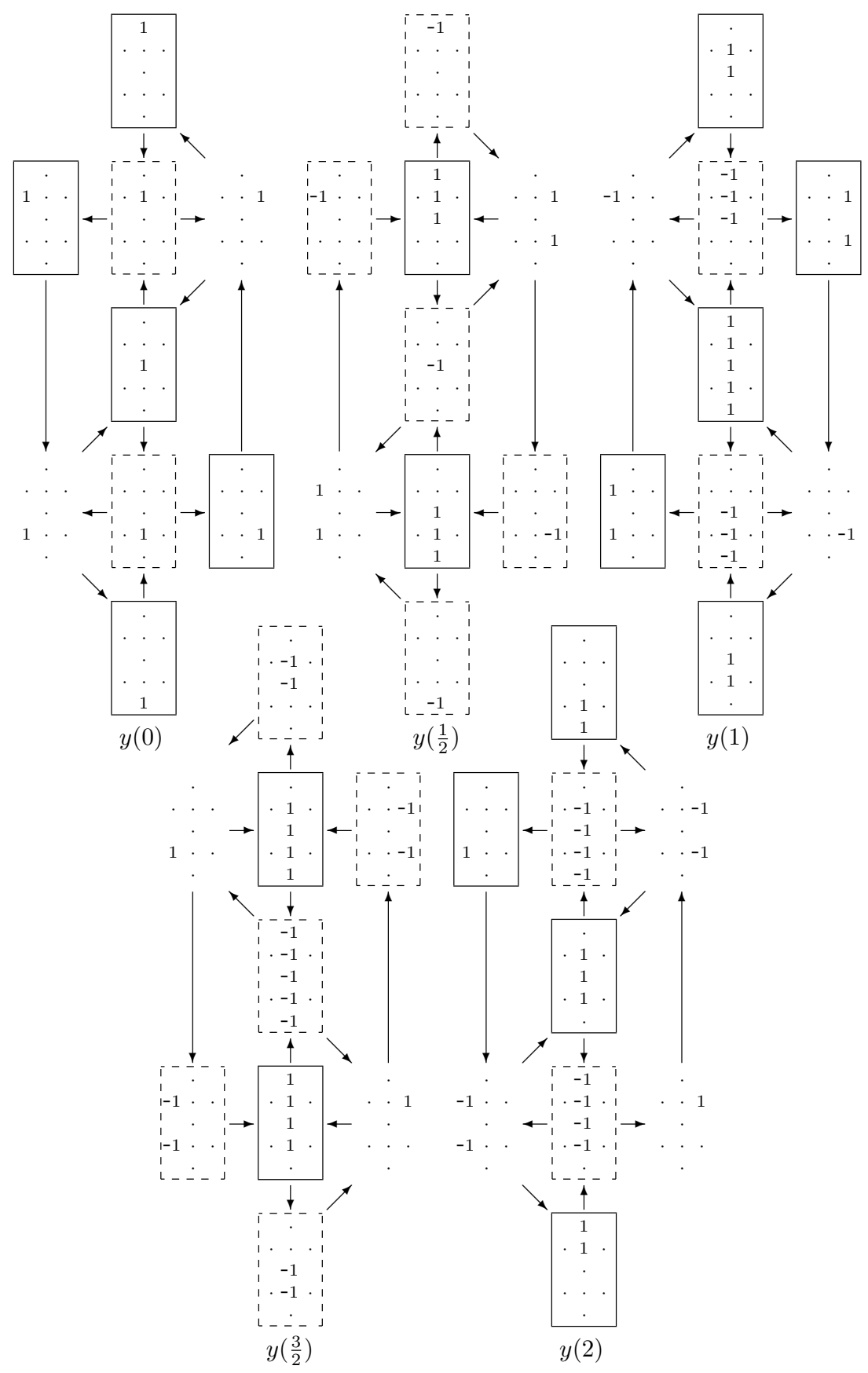

Figure 8. Tropical Y-system of type $B_{2}$ at level 3 in the region $0 \leq u \leq 2$. 
given explicitly. It is better to view it in the forward direction from $u=0$ to 2 . We observe that no column affects any other in mutations. More precisely, the first and third columns (from the left) are in the same mutation pattern as the tropical Y-system of type $A_{2}$ studied in [FZ2, FZ3] (see also [Nkn] for more details). The second column is in the same mutation pattern as the tropical Y-system of type $A_{5}$ (at the twice faster pace). By [FZ3, Proposition 10.7], $\left[y_{\mathbf{i}}(u)\right]_{\mathbf{T}}$ for $(\mathbf{i}, u): \mathbf{p}_{+}$ are positive in the region $0 \leq u<3$ (3 is the Coxeter number of $A_{2}$ and half of the Coxeter number of $A_{5}$ ). The factorization occurs for the same reason as before; at any mutation point $(\mathbf{i}, u): \mathbf{p}_{+}$the arrows between adjacent columns are incoming while $\left[y_{\mathbf{i}}(u)\right]_{\mathbf{T}}$ are positive. Therefore, these arrows can be forgotten, and we have (ii). Finally, (iii) is also a consequence of [FZ3, Proposition 10.7]. Again, this argument is also applicable to any rank $r$ and level $\ell$ by replacing the number 3 in the above with $\ell$, which is the Coxeter number of $A_{\ell-1}$ and half of the Coxeter number of $A_{2 \ell-1}$.

We obtain two important corollaries of Propositions 3.2 and 4.1 .

Theorem 4.2. For $\left[\mathcal{G}_{Y}(B, y)\right]_{\mathbf{T}}$ with $B=B_{\ell}\left(B_{r}\right)$, the following relations hold:

(i) Half periodicity: $\left[y_{\mathbf{i}}\left(u+h^{\vee}+\ell\right)\right]_{\mathbf{T}}=\left[y_{\boldsymbol{\omega}(\mathbf{i})}(u)\right]_{\mathbf{T}}$.

(ii) Full periodicity: $\left[y_{\mathbf{i}}\left(u+2\left(h^{\vee}+\ell\right)\right)\right]_{\mathbf{T}}=\left[y_{\mathbf{i}}(u)\right]_{\mathbf{T}}$.

Proof. (i) follows from (iii) and (iv) of Propositions 3.2 and 4.1; and (ii) follows from (i).

We remark that the half periodicity above is compatible with the one for mutation matrices; namely, set $B(u)=B:=B_{\ell}\left(B_{r}\right),-B, \boldsymbol{r}(B),-\boldsymbol{r}(B)$ for $u \equiv$ $0, \frac{1}{2}, 1, \frac{3}{2} \bmod 2 \mathbb{Z}$, respectively. Then

$$
B\left(u+h^{\vee}+\ell\right)=\boldsymbol{\omega}(B(u))
$$

due to Lemma 2.1(ii).

Theorem 4.3. For $\left[\mathcal{G}_{Y}(B, y)\right]_{\mathbf{T}}$ with $B=B_{\ell}\left(B_{r}\right)$, let $N_{+}$and $N_{-}$denote the total numbers of positive and negative monomials, respectively, among $\left[y_{\mathbf{i}}(u)\right]_{\mathbf{T}}$ for $(\mathbf{i}, u): \mathbf{p}_{+}$in the region $0 \leq u<2\left(h^{\vee}+\ell\right)$. Then

$$
N_{+}=2 \ell(\ell r+\ell-1), \quad N_{-}=2 r(2 \ell r-2 r+1) .
$$

Proof. This follows from (i) and (ii) of Propositions 3.2 and 4.1, and (i) in Theorem 4.2. 


\section{§5. 2-Calabi-Yau realization and periodicity}

In this section we prove Theorems 1.5 and 1.6. Our method is based on the 2Calabi-Yau realizations of cluster algebras. They are triangulated categories satisfying the 2-Calabi-Yau property, and their categorical structure realizes the combinatorial structure of cluster algebras.

\section{$\S 5.1$. Periodicity theorem for cluster algebras}

In this subsection we assume that $B=\left(B_{i j}\right)_{i, j \in I}$ is an arbitrary skew-symmetric (integer) matrix. Let $\mathcal{A}(B, x, y)$ be the cluster algebra with coefficients in the universal semifield $\mathbb{Q}_{\mathrm{sf}}(y)$, where $(B, x, y)$ is the initial seed. Alternatively, we may consider the cluster pattern assigned to the $n$-regular tree $\mathbb{T}_{n}, n=|I|$, with the following data (see Section 2.1). Let $Q$ be the quiver (without loops or 2cycles) corresponding to $B$ with the set of vertices $I$. We fix a vertex $t_{0} \in \mathbb{T}_{n}$, and assign the initial seed to $t_{0},\left(Q\left(t_{0}\right), x\left(t_{0}\right), y\left(t_{0}\right)\right)=(Q, x, y)$. Then we have a seed $(Q(t), x(t), y(t))$ for each $t \in \mathbb{T}_{n}$, where we identify the quiver $Q(t)$ and the corresponding skew-symmetric matrix $B(t)$ in each seed.

Let $\operatorname{Trop}(y)$ be the tropical semifield. As in Section 3.1, we define the tropical evaluation of $y_{i}(t)$,

$$
\left[y_{i}(t)\right]_{\mathbf{T}} \in \operatorname{Trop}(y)
$$

as the image of $y_{i}(t) \in \mathbb{Q}_{\text {sf }}(y)$ under the natural map $\pi_{\mathbf{T}}: \mathbb{Q}_{\mathbf{s f}}(y) \rightarrow \operatorname{Trop}(y)$. Here we call $\left[y_{i}(t)\right]_{\mathbf{T}}$ 's the principal coefficients in accordance with the nomenclature of [FZ3]. (They may also be called the tropical $Y$-variables.)

For an automorphism $\omega: I \rightarrow I$, we define a new quiver $\omega(Q)$ with the same set $I$ of vertices by drawing an arrow $\omega(a): \omega(i) \rightarrow \omega(j)$ in $\omega(Q)$ for each arrow $a: i \rightarrow j$ in $Q$.

The following theorem is Observation 1 explained in Section 1.5. Just as it is crucial in our proof of the periodicities of the T-systems and Y-systems in this paper and also in [IIKKN], it is expected to be useful in other applications.

Theorem 5.1 (Periodicity theorem). Let $B$ be an arbitrary skew-symmetric matrix, and let $Q$ be the quiver corresponding to B. Let $(Q(t), x(t), y(t))$ be the seed at $t \in \mathbb{T}_{n}$ for $\mathcal{A}(B, x, y)$ as above. Suppose that there exists some $t \in \mathbb{T}_{n}$ and an automorphism $\omega$ of $I$ such that $\left[y_{i}(t)\right]_{\mathbf{T}}=\left[y_{\omega(i)}\right]_{\mathbf{T}}$ for any $i \in I$. Then

$$
\begin{aligned}
Q(t) & =\omega^{-1}(Q) \quad\left(\text { equivalently, } B_{i j}(t)=B_{\omega(i) \omega(j)}\right), \\
x_{i}(t) & =x_{\omega(i)} \quad(i \in I), \\
y_{i}(t) & =y_{\omega(i)} \quad(i \in I) .
\end{aligned}
$$


In particular, the periodicity of seeds of $\mathcal{A}(B, x, y)$ coincides with the periodicity of principal coefficient tuples.

Let $\mathcal{A}_{\bullet}(B, x, y)$ be the cluster algebra with coefficients in the tropical semifield $\operatorname{Trop}(y)$, where $(B, x, y)$ is the initial seed. It is also called the cluster algebra with principal coefficients [FZ3]. Note that a coefficient $y_{i}(t)$ in $\mathcal{A}_{\bullet}(B, x, y)$ coincides with $\left[y_{i}(t)\right]_{\mathbf{T}}$ in $(5.1)$.

According to [FZ3, Theorem 4.6] and its proof, Theorem 5.1 reduces to the following result for $\mathcal{A}_{\bullet}(B, x, y)$.

Theorem 5.2. Let $B$ and $Q$ be as in Theorem 5.1. Let $(Q(t), x(t), y(t))$ be the seed at $t \in \mathbb{T}_{n}$ for $\mathcal{A}_{\bullet}(B, x, y)$. Suppose that there exists some $t \in \mathbb{T}_{n}$ and an automorphism $\omega$ of $I$ such that $y_{i}(t)=y_{\omega(i)}$ for any $i \in I$. Then

$$
Q(t)=\omega^{-1}(Q), \quad x_{i}(t)=x_{\omega(i)} \quad(i \in I) .
$$

We give a proof of Theorem 5.2 in Section 5.3.

Remark 5.3. Theorem 5.2 was essentially conjectured by Fomin-Zelevinsky [FZ3, Conjecture 4.7] for an arbitrary skew-symmetrizable matrix $B$. Therefore, we partly prove the conjecture for a skew-symmetric matrix $B$. Note that our claim is a little stronger than [FZ3, Conjecture 4.7] because the periodicity of the principal parts of exchange matrices also follows from the periodicity of principal coefficients. (Meanwhile, the periodicity of the complementary parts of exchange matrices coincides with the periodicity of principal coefficients by definition [FZ3].)

Remark 5.4. Since Theorems 5.1 and 5.2 involve quivers corresponding to arbitrary skew-symmetric matrices, the categories appearing in their proof will in general have infinite-dimensional morphism spaces. It is of interest whether the quivers which appear in our applications of the two theorems actually admit categorifications by Hom-finite 2-CY categories. We conjecture that this is indeed the case for all levels $l \geq 2$. For $l=2$, it is clear since the quiver $Q_{2}\left(B_{r}\right)$ is of finite cluster type $D_{2 r+1}$. However, for $l>2$, the conjecture appears to be nontrivial.

\section{$\S 5.2$ 2-Calabi-Yau realization of $\mathcal{A}_{\bullet}(B, x, y)$}

Our proof of Theorem 5.2 uses the categorification of the cluster algebra $\mathcal{A}_{\bullet}(B, x, y)$ by a certain 2-Calabi-Yau category [Pa, FK, Ke1, A, KY, Pl1, Pl2]. Here we review the recent result by Plamondon [Pl1, Pl2].

Let $Q$ be the quiver corresponding to an arbitrary skew-symmetric matrix $B$. Define the principal extension $\widetilde{Q}$ of $Q$ as the quiver obtained from $Q$ by adding a new vertex $i^{\prime}$ and an arrow $i^{\prime} \rightarrow i$ for each $i \in I$. Thus the set of vertices in $\widetilde{Q}$ 
is given by $\widetilde{I}:=I \sqcup I^{\prime}$ with $I^{\prime}:=\left\{i^{\prime} \mid i \in I\right\}$. By mutations one can associate a quiver $\widetilde{Q}(t)$ with each $t \in \mathbb{T}_{n}$, where $\widetilde{Q}(t)$ contains $Q(t)$ as a full subquiver. Note that we do not make mutations for 'frozen indices' $i^{\prime} \in I^{\prime}$.

We fix a base field $K$ to be an infinite one. Since $\widetilde{Q}$ does not have loops or 2-cycles, we have the following result by [DWZ1, Corollary 7.4].

Proposition 5.5. There exists a nondegenerate potential $W$ on $\widetilde{Q}$.

From now on we assume $W$ is a nondegenerate potential on $\widetilde{Q}$. We denote by

$$
\mathcal{C}:=\mathcal{C}_{(\widetilde{Q}, W)}
$$

the cluster category associated to the quiver with potential $(\widetilde{Q}, W)$, which is not necessarily Hom-finite [A, KY, Pl1]. The category $\mathcal{C}$ canonically contains a rigid object

$$
T=\bigoplus_{i \in \widetilde{I}} T_{i} \in \mathcal{C}
$$

such that $\operatorname{End}_{\mathcal{C}}(T)$ is isomorphic to the Jacobian algebra of $(\widetilde{Q}, W)$. For each $t \in \mathbb{T}_{n}$, we have a rigid object

$$
T(t)=\bigoplus_{i \in \widetilde{I}} T_{i}(t) \in \mathcal{C}
$$

by applying successive mutations (see [Pl1, Section 2.6]). We have $T_{i^{\prime}}(t)=T_{i^{\prime}}$ for any $i^{\prime} \in I^{\prime}$.

From the definition of $T(t)$ and the nondegeneracy of $(\widetilde{Q}, W)$ we have the following description of $\widetilde{Q}(t)$.

Proposition 5.6. For each $t \in \mathbb{T}_{n}$, the quiver of $\operatorname{End}_{\mathcal{C}}(T(t))$ is $\widetilde{Q}(t)$, where each vertex $i \in \widetilde{I}$ corresponds to the direct summand $T_{i}(t)$ of $T(t)$.

As usual we denote by add $T(t)$ the full subcategory of $\mathcal{C}$ consisting of all direct summands of finite direct sums of copies of $T(t)$. We denote by

$$
\operatorname{pr} T(t)
$$

the full subcategory of $\mathcal{C}$ consisting of all objects $M \in \mathcal{C}$ such that there exists a triangle

$$
T^{\prime \prime} \rightarrow T^{\prime} \rightarrow M \rightarrow T^{\prime \prime}[1]
$$

in $\mathcal{C}$ with $T^{\prime}, T^{\prime \prime} \in \operatorname{add} T(t)$. 
Proposition 5.7 ([Pl1, Proposition 2.7, Corollary 2.12]). (1) $\operatorname{pr} T(t)=\operatorname{pr} T$ for any $t \in \mathbb{T}_{n}$.

(2) The category $\operatorname{pr} T$ is Krull-Schmidt in the sense that any object can be written as a finite direct sum of objects whose endomorphism rings are local.

Now let us introduce the following notion.

Definition 5.8. Let $t \in \mathbb{T}_{n}$. For an object $T^{\prime}=\bigoplus_{i \in \widetilde{I}} T_{i}(t)^{\ell_{i}}$ in add $T(t)$, we put

$$
\left[T^{\prime}\right]_{T(t)}:=\left(\ell_{i}\right)_{i \in \widetilde{I}} \in \mathbb{Z}^{\widetilde{I}} .
$$

For an object $M \in \operatorname{pr} T(t)$, we take a triangle (5.8) and define the index of $M$ by

$$
\operatorname{ind}_{T(t)}(M):=\left[T^{\prime}\right]_{T(t)}-\left[T^{\prime \prime}\right]_{T(t)} \in \mathbb{Z}^{\widetilde{I}} .
$$

This is independent of the choice of the triangle (5.8) by Proposition 5.7(2).

We have the following relationship between indices and principal coefficients.

Proposition 5.9 ([Pl2, Corollary 3.10], [Ke1, Theorem 7.13(b)]). Let $y_{i}(t)$ be a coefficient in $\mathcal{A}_{\bullet}(B, x, y)$. For $t \in \mathbb{T}_{n}$, we put $y_{j}(t)=\prod_{i \in I} y_{i}^{c_{i j}(t)}$ for any $j \in I$. Then

$$
-\operatorname{ind}_{T(t)}\left(T_{i}[1]\right)=\left(c_{i j}(t)\right)_{j \in I} \in \mathbb{Z}^{I}
$$

for any $i \in I$, where we embed $\mathbb{Z}^{I}$ into $\mathbb{Z}^{\widetilde{I}}$ naturally.

Remark 5.10. Proposition 5.9 shows that the principal coefficients of a quiver $Q$ are determined by the $g$-vectors of the opposite quiver $Q^{\mathrm{op}}$. This can also be deduced from Conjecture 1.6 (proved in Theorem 1.7) of [DWZ2] using Remark 7.15 of [FZ3] on 'Langlands duality'.

The following analogue of [DK, Theorem 2.3] is an important ingredient in our proof.

Proposition 5.11 ([Pl2, Proposition 3.1]). Let $X, Y \in \operatorname{pr} T$ be rigid objects and $t \in \mathbb{T}_{n}$. Then $X \simeq Y$ if and only if $\operatorname{ind}_{T(t)}(X)=\operatorname{ind}_{T(t)}(Y)$.

Let us introduce a Caldero-Chapoton-type map.

Definition 5.12. Define a full subcategory

$$
\mathcal{D}:=\left\{M \in \operatorname{pr} T \cap \operatorname{pr} T[-1] \mid \operatorname{dim}_{K} \operatorname{Hom}_{\mathcal{C}}(T, M[1])<\infty\right\} .
$$


For any object $M \in \mathcal{D}$, we define an element $\mathbb{X}_{M}$ in $\mathbb{Z}\left[x^{ \pm 1}, y\right]$ by

$$
\text { (5.14) } \quad \mathbb{X}_{M}:=\left(\prod_{i \in I} x_{i}^{\operatorname{ind}_{T}(M)_{i}} y_{i}^{\operatorname{ind}_{T}(M)_{i^{\prime}}}\right) \sum_{e \in \mathbb{Z}^{I}} \chi\left(\operatorname{Gr}_{e}\left(\operatorname{Hom}_{\mathcal{C}}(T, M[1])\right)\right) \prod_{j \in I} \widehat{y}_{j}^{e_{j}}
$$

where $\mathrm{Gr}_{e}$ is the quiver Grassmannian and $\chi$ is the Euler characteristic.

For each $t \in \mathbb{T}_{n}$ we have $T(t) \in \mathcal{D}$ by Proposition 5.7(1). The following description of the cluster variables in $\mathcal{A}_{\bullet}(B, x, y)$ is crucial in our proof.

Proposition 5.13. Let $x_{i}(t)$ be a cluster variable in $\mathcal{A}_{\bullet}(B, x, y)$. Then

$$
x_{i}(t)=\mathbb{X}_{T_{i}(t)}
$$

for any $t \in \mathbb{T}_{n}$ and $i \in I$.

Proof. Specializing $(B, n)$ in $[\mathrm{Pl1}$, Theorem 3.12] to

$$
\left(\left(\begin{array}{cc}
B & -E_{n} \\
E_{n} & O
\end{array}\right), 2 n\right)
$$

we find that $\mathbb{X}$ is a cluster character in the sense of [Pl1, Definition 3.10]. Now the assertion is an immediate consequence.

\section{§5.3. Proof of Theorem 5.2}

Now we are ready to prove Theorem 5.2. Since $y_{j}(t)=y_{\omega(j)}$ for any $j \in I$, in Proposition 5.9 we have

$$
c_{i j}(t)= \begin{cases}1, & i=\omega(j) \\ 0, & \text { otherwise }\end{cases}
$$

for any $i, j \in I$. Thus $\operatorname{ind}_{T(t)}\left(T_{\omega(j)}[1]\right)=\operatorname{ind}_{T(t)}\left(T_{j}(t)[1]\right)$ for any $j \in I$. By Proposition 5.11,

$$
T_{\omega(j)} \simeq T_{j}(t)
$$

for any $j \in I$. By Proposition 5.13 and (5.18), we have

$$
x_{j}(t)=\mathbb{X}_{T_{j}(t)}=\mathbb{X}_{T_{\omega(j)}}=x_{\omega(j)}
$$

for any $j \in I$.

Finally, by (5.18) and Proposition 5.6,

$$
\omega^{-1}(\widetilde{Q})=\widetilde{Q}(t) \text { and so } \omega^{-1}(Q)=Q(t) .
$$




\section{§5.4. Proof of periodicities of T-systems and Y-systems}

Now the proof of Theorems 1.5 and 1.6 is at hand.

As corollaries of Theorems 4.2 and 5.1 we immediately obtain the periodicities of the cluster variables and coefficients in $\mathcal{A}(B, x, y)$ with $B=B_{\ell}\left(B_{r}\right)$.

Corollary 5.14. For $\mathcal{A}(B, x, y)$ with $B=B_{\ell}\left(B_{r}\right)$, the following relations hold:

(i) Half periodicity: $x_{\mathbf{i}}\left(u+h^{\vee}+\ell\right)=x_{\boldsymbol{\omega}(\mathbf{i})}(u)$.

(ii) Full periodicity: $x_{\mathbf{i}}\left(u+2\left(h^{\vee}+\ell\right)\right)=x_{\mathbf{i}}(u)$.

Corollary 5.15. For $\mathcal{G}(B, y)$ with $B=B_{\ell}\left(B_{r}\right)$, the following relations hold:

(i) Half periodicity: $y_{\mathbf{i}}\left(u+h^{\vee}+\ell\right)=y_{\boldsymbol{\omega}(\mathbf{i})}(u)$.

(ii) Full periodicity: $y_{\mathbf{i}}\left(u+2\left(h^{\vee}+\ell\right)\right)=y_{\mathbf{i}}(u)$.

As further corollaries of Corollaries 5.14 and 5.15 and Theorems 2.7 and 2.10 we obtain Theorems 1.5 and 1.6.

As a corollary of Theorems 4.2 and 5.2 we also obtain the periodicity of $F$ polynomials [FZ3] (see Section 2.1 for the definition), which will be used in the next section.

Corollary 5.16. For $\mathcal{A}(B, x, y)$ with $B=B_{\ell}\left(B_{r}\right)$, let $F_{\mathbf{i}}(u)$ be the $F$-polynomial at $(\mathbf{i}, u)$. Then the following relations hold:

(i) Half periodicity: $F_{\mathbf{i}}\left(u+h^{\vee}+\ell\right)=F_{\boldsymbol{\omega}(\mathbf{i})}(u)$.

(ii) Full periodicity: $F_{\mathbf{i}}\left(u+2\left(h^{\vee}+\ell\right)\right)=F_{\mathbf{i}}(u)$.

\section{§6. Dilogarithm identities}

In this section we prove Theorem 1.10. In the cluster algebraic formulation here, Theorem 1.10 is expressed as follows.

Theorem 6.1. For $\mathcal{G}_{Y}(B, y)$ with $B=B_{\ell}\left(B_{r}\right)$, let $y_{m}^{(a)}(u)$ be the coefficient tuple in Theorem 2.10. Then, for any semifield homomorphism $\varphi: \mathbb{Q}_{\mathrm{sf}}(y) \rightarrow \mathbb{R}_{+}$,

$$
\frac{6}{\pi^{2}} \sum_{(a, m, u) \in S_{+}^{\prime}} L\left(\frac{\varphi\left(y_{m}^{(a)}(u)\right)}{1+\varphi\left(y_{m}^{(a)}(u)\right)}\right)=2 r(2 r \ell-2 r+1),
$$

where $S_{+}^{\prime}=\left\{(a, m, u) \in \mathcal{I}_{\ell+}^{\prime} \mid 0 \leq u<2\left(h^{\vee}+\ell\right)\right\}$.

Let $F_{m}^{(a)}(u)$ denote the $F$-polynomial $F_{\mathbf{i}}(v)$ at $(\mathbf{i}, v)=g((a, m, u))$, i.e., with the same parametrization by $\mathcal{I}_{\ell+}$ as $x_{m}^{(a)}(u)$. 
Lemma 6.2. (i) For $(a, m, u) \in \mathcal{I}_{\ell+}^{\prime}$, the following relations hold:

$$
\begin{gathered}
F_{m}^{(a)}\left(u-\frac{1}{t_{a}}\right) F_{m}^{(a)}\left(u+\frac{1}{t_{a}}\right)=\left[\frac{y_{m}^{(a)}(u)}{1+y_{m}^{(a)}(u)}\right]_{\mathbf{T}} \prod_{(b, k, v) \in \mathcal{I}_{\ell+}} F_{k}^{(b)}(v)^{G(b, k, v ; a, m, u)} \\
+\left[\frac{1}{1+y_{m}^{(a)}(u)}\right]_{\mathbf{T}} F_{m-1}^{(a)}(u) F_{m+1}^{(a)}(u), \\
\begin{aligned}
y_{m}^{(a)}(u)=\left[y_{m}^{(a)}(u)\right]_{\mathbf{T}} \frac{\prod_{(b, k, v) \in \mathcal{I}_{\ell+}} F_{k}^{(b)}(v)^{G(b, k, v ; a, m, u)}}{F_{m-1}^{(a)}(u) F_{m+1}^{(a)}(u)} \\
1+y_{m}^{(a)}(u)=\left[1+y_{m}^{(a)}(u)\right]_{\mathbf{T}} \frac{F_{m}^{(a)}\left(u-\frac{1}{t_{a}}\right) F_{m}^{(a)}\left(u+\frac{1}{t_{a}}\right)}{F_{m-1}^{(a)}(u) F_{m+1}^{(a)}(u)}
\end{aligned}
\end{gathered}
$$

(ii) Periodicity: $F_{m}^{(a)}\left(u+2\left(h^{\vee}+\ell\right)\right)=F_{m}^{(a)}(u)$.

(iii) Each polynomial $F_{m}^{(a)}(u)$ has constant term 1.

Proof. (i) (6.2) is a specialization of (2.4); (6.3) is due to [FZ3, Proposition 3.13]; and (6.4) follows from (6.2) and (6.3).

(ii) This is a special case of Corollary 5.16.

(iii) The claim is shown by induction on $u$, by using $F_{\mathbf{i}}(0)=1,(6.2)$, and Proposition 4.1 (cf. [FZ3, Proposition 5.6]).

According to [FS, C, Nkn], the proof of Theorem 1.10 reduces to the next claim.

Proposition 6.3. (i) In $\bigwedge^{2} \mathbb{Q}_{\mathrm{sf}}(y)$, we have

$$
\sum_{(a, m, u) \in S_{+}^{\prime}} y_{m}^{(a)}(u) \wedge\left(1+y_{m}^{(a)}(u)\right)=0 .
$$

(ii) The total number of negative monomials among $\left[y_{m}^{(a)}(u)\right]_{\mathbf{T}}\left((a, m, u) \in S_{+}^{\prime}\right)$ is $2 r(2 r \ell-2 r+1)$.

(ii) is already proved in Theorem 4.3. Let us prove (i). The argument is parallel to the simply laced case [Nkn, Proposition 4.1], but a little more complicated. Therefore, we present the calculations.

We put (6.3) and (6.4) into (6.5), and expand it.

Firstly,

$$
\sum_{(a, m, u) \in S_{+}^{\prime}}\left[y_{m}^{(a)}(u)\right]_{\mathbf{T}} \wedge\left[1+y_{m}^{(a)}(u)\right]_{\mathbf{T}}=0
$$

since each monomial $\left[y_{m}^{(a)}(u)\right]_{\mathbf{T}}$ is either positive or negative by Proposition 4.1. 
Secondly, the contributions from the terms involving only $F_{\mathbf{i}}(u)$ 's vanish. To see this, we divide them into two parts. The first part

$$
\sum_{(a, m, u) \in S_{+}^{\prime}} F_{m-1}^{(a)}(u) F_{m+1}^{(a)}(u) \wedge F_{m}^{(a)}\left(u-\frac{1}{t_{a}}\right) F_{m}^{(a)}\left(u+\frac{1}{t_{a}}\right)
$$

vanishes due to the symmetry argument of [CGT, Section 3], where we use the periodicity of $F$-polynomials (Lemma 6.2(ii)). The second part

$$
\sum_{(a, m, u) \in S_{+}^{\prime}} \prod_{(b, k, v) \in \mathcal{I}_{\ell+}} F_{k}^{(b)}(v)^{G(b, k, v ; a, m, u)} \wedge \frac{F_{m}^{(a)}\left(u-\frac{1}{t_{a}}\right) F_{m}^{(a)}\left(u+\frac{1}{t_{a}}\right)}{F_{m-1}^{(a)}(u) F_{m+1}^{(a)}(u)}
$$

reduces, by the symmetry argument again, to the sum consisting of all terms with $(a, b)=(r-1, r),(r, r-1) ;$ namely,

$$
\begin{aligned}
& \sum_{m=1}^{\ell-1} \sum_{\substack{u \equiv 0 \bmod \mathbb{Z} \\
0 \leq u<2\left(h^{\vee}+\ell\right)}} F_{2 m}^{(r)}(u) \wedge \frac{F_{m}^{(r-1)}(u-1) F_{m}^{(r-1)}(u+1)}{F_{m-1}^{(r-1)}(u) F_{m+1}^{(r-1)}(u)} \\
& +\sum_{m=1}^{\ell-1} \sum_{\substack{u \equiv \frac{1}{2} \bmod \mathbb{Z} \\
0 \leq u<2\left(h^{\vee}+\ell\right)}} F_{m}^{(r-1)}\left(u-\frac{1}{2}\right) F_{m}^{(r-1)}\left(u+\frac{1}{2}\right) \wedge \frac{F_{2 m}^{(r)}\left(u-\frac{1}{2}\right) F_{2 m}^{(r)}\left(u+\frac{1}{2}\right)}{F_{2 m-1}^{(r)}(u) F_{2 m+1}^{(r)}(u)} \\
& +\sum_{m=0}^{\ell-1} \sum_{\substack{u \equiv 0 \bmod \mathbb{Z} \\
0 \leq u<2\left(h^{\vee}+\ell\right)}} F_{m}^{(r-1)}(u) F_{m+1}^{(r-1)}(u) \wedge \frac{F_{2 m+1}^{(r)}\left(u-\frac{1}{2}\right) F_{2 m+1}^{(r)}\left(u+\frac{1}{2}\right)}{F_{2 m}^{(r)}(u) F_{2 m+2}^{(r)}(u)},
\end{aligned}
$$

where $F_{0}^{(r-1)}(u)=F_{\ell}^{(r-1)}(u)=F_{0}^{(r)}(u)=F_{2 \ell}^{(r)}(u)=1$. It is easy to check that all the terms cancel each other.

Thirdly, the contributions from the remaining terms are as follows, where $S_{+}=\left\{(a, m, u) \in \mathcal{I}_{\ell+} \mid 0 \leq u<2\left(h^{\vee}+\ell\right)\right\}:$

$$
\begin{gathered}
\sum_{(a, m, u) \in S_{+}^{\prime}}\left[y_{m}^{(a)}(u)\right]_{\mathbf{T}} \wedge F_{m}^{(a)}\left(u-\frac{1}{t_{a}}\right) F_{m}^{(a)}\left(u+\frac{1}{t_{a}}\right) \\
=\sum_{(a, m, u) \in S_{+}}\left[y_{m}^{(a)}\left(u-\frac{1}{t_{a}}\right)\right]_{\mathbf{T}}\left[y_{m}^{(a)}\left(u+\frac{1}{t_{a}}\right)\right]_{\mathbf{T}} \wedge F_{m}^{(a)}(u), \\
-\sum_{(a, m, u) \in S_{+}^{\prime}}\left[y_{m}^{(a)}(u)\right]_{\mathbf{T}} \wedge F_{m-1}^{(a)}(u) F_{m+1}^{(a)}(u) \\
=-\sum_{(a, m, u) \in S_{+}}\left[y_{m-1}^{(a)}(u)\right]_{\mathbf{T}}\left[y_{m+1}^{(a)}(u)\right]_{\mathbf{T}} \wedge F_{m}^{(a)}(u)
\end{gathered}
$$




$$
\begin{gathered}
\sum_{(a, m, u) \in S_{+}^{\prime}}\left[1+y_{m}^{(a)}(u)\right]_{\mathbf{T}} \wedge F_{m-1}^{(a)}(u) F_{m+1}^{(a)}(u) \\
=\sum_{(a, m, u) \in S_{+}}\left[1+y_{m-1}^{(a)}(u)\right]_{\mathbf{T}}\left[1+y_{m+1}^{(a)}(u)\right]_{\mathbf{T}} \wedge F_{m}^{(a)}(u), \\
-\sum_{(a, m, u) \in S_{+}^{\prime}}\left[1+y_{m}^{(a)}(u)\right]_{\mathbf{T}} \wedge \prod_{(b, k, v) \in \mathcal{I}_{\ell+}} F_{k}^{(b)}(v)^{G(b, k, v ; a, m, u)} \\
=-\sum_{(a, m, u) \in S_{+}} \prod_{(b, k, v) \in \mathcal{I}_{\ell+}^{\prime}}\left[1+y_{k}^{(b)}(v)\right]_{\mathbf{T}}^{G(a, m, u ; b, k, v)} \wedge F_{m}^{(a)}(u) .
\end{gathered}
$$

These terms cancel if we have the relation

$$
\left[y_{m}^{(a)}\left(u-\frac{1}{t_{a}}\right)\right]_{\mathbf{T}}\left[y_{m}^{(a)}\left(u+\frac{1}{t_{a}}\right)\right]_{\mathbf{T}}=\frac{\prod_{(b, k, v) \in \mathcal{I}_{\ell+}^{\prime}}\left[1+y_{k}^{(b)}(v)\right]_{\mathbf{T}}^{t} G(b, k, v ; a, m, u)}{\left[1+y_{m-1}^{(a)}(u)^{-1}\right]_{\mathbf{T}}\left[1+y_{m+1}^{(a)}(u)^{-1}\right]_{\mathbf{T}}} .
$$

This is nothing but the Y-system (1.6), therefore, it holds by Lemma 2.8.

This completes the proof of Proposition 6.3.

\section{§7. Alternative proof of periodicities of T-systems and Y-systems of simply laced type}

Let $\left(X_{r}, X_{r^{\prime}}^{\prime}\right)$ be a pair of simply laced Dynkin diagrams of finite type with index sets $I$ and $I^{\prime}$.

As an application of Theorem 5.1, we give an alternative and simplified proof of the periodicities of the T-systems and Y-systems associated with $\left(X_{r}, X_{r^{\prime}}^{\prime}\right)$. They were formerly proved in [FZ1, FZ2] for the $X_{r^{\prime}}^{\prime}=A_{1}$ ('level 2 case') and in [Ke1, IIKNS, Ke2] for the general case.

For a family of variables $\left\{T_{i i^{\prime}}(u) \mid i \in I, i^{\prime} \in I^{\prime}, u \in \mathbb{Z}\right\}$, the $T$-system $\mathbb{T}\left(X_{r}, X_{r^{\prime}}^{\prime}\right)$ associated with a pair $\left(X_{r}, X_{r^{\prime}}^{\prime}\right)$ is the system of the relations

$$
T_{i i^{\prime}}(u-1) T_{i i^{\prime}}(u+1)=\prod_{j: j \sim i} T_{j i^{\prime}}(u)+\prod_{j^{\prime}: j^{\prime} \sim i^{\prime}} T_{i j^{\prime}}(u),
$$

where $j \sim i$ means $j$ is adjacent to $i$ in $X_{r}$, while $j^{\prime} \sim i^{\prime}$ means $j^{\prime}$ is adjacent to $i^{\prime}$ in $X_{r^{\prime}}^{\prime}$.

For a family of variables $\left\{Y_{i i^{\prime}}(u) \mid i \in I, i^{\prime} \in I^{\prime}, u \in \mathbb{Z}\right\}$, the $Y$-system $\mathbb{Y}\left(X_{r}, X_{r^{\prime}}^{\prime}\right)$ associated with a pair $\left(X_{r}, X_{r^{\prime}}^{\prime}\right)$ is the system of relations

$$
Y_{i i^{\prime}}(u-1) Y_{i i^{\prime}}(u+1)=\frac{\prod_{j: j \sim i}\left(1+Y_{j i^{\prime}}(u)\right)}{\prod_{j^{\prime}: j^{\prime} \sim i^{\prime}}\left(1+Y_{i j^{\prime}}(u)^{-1}\right)} .
$$

Let $C=\left(C_{i j}\right)_{i, j \in I}$ and $C^{\prime}=\left(C_{i^{\prime} j^{\prime}}\right)_{i^{\prime}, j^{\prime} \in I}$ be a pair of Cartan matrices of types $X_{r}$ and $X_{r^{\prime}}^{\prime}$ with fixed bipartite decompositions $I=I_{+} \sqcup I_{-}$and $I^{\prime}=I_{+}^{\prime} \sqcup I_{-}^{\prime}$. 
Set $\mathbf{I}=I \times I^{\prime}$. For $\mathbf{i}=\left(i, i^{\prime}\right) \in \mathbf{I}$, let us write $\mathbf{i}:(++)$ if $\left(i, i^{\prime}\right) \in I_{+} \times I_{+}^{\prime}$, etc. Define the matrix $B=B\left(X_{r}, X_{r^{\prime}}^{\prime}\right)=\left(B_{\mathbf{i} \mathbf{j}}\right)_{\mathbf{i}, \mathbf{j} \in \mathbf{I}}$ by

$$
B_{\mathbf{i j}}= \begin{cases}-C_{i j} \delta_{i^{\prime} j^{\prime}}, & \mathbf{i}:(-+), \mathbf{j}:(++) \text { or } \mathbf{i}:(+-), \mathbf{j}:(--), \\ C_{i j} \delta_{i^{\prime} j^{\prime}}, & \mathbf{i}:(++), \mathbf{j}:(-+) \text { or } \mathbf{i}:(--), \mathbf{j}:(+-), \\ -\delta_{i j} C_{i^{\prime} j^{\prime}}^{\prime}, & \mathbf{i}:(++), \mathbf{j}:(+-) \text { or } \mathbf{i}:(--), \mathbf{j}:(-+), \\ \delta_{i j} C_{i^{\prime} j^{\prime}}^{\prime}, & \mathbf{i}:(+-), \mathbf{j}:(++) \text { or } \mathbf{i}:(-+), \mathbf{j}:(--), \\ 0, & \text { otherwise. }\end{cases}
$$

Then, as in Section 2, one can express the T-systems and Y-systems in terms of the cluster algebra $\mathcal{A}(B, x, y)$ and its coefficient group $\mathcal{G}(B, y)$ with $B=B\left(X_{r}, X_{r^{\prime}}^{\prime}\right)$ (cf. [Nkn, Proposition 2.6]).

Theorem 7.1. The following relations hold for the tropical $Y$-system of $\mathcal{G}(B, y)$ with $B=B\left(X_{r}, X_{r^{\prime}}^{\prime}\right)$ :

(i) Half periodicity: $\left[y_{\mathbf{i}}\left(u+h+h^{\prime}\right)\right]_{\mathbf{T}}=\left[y_{\boldsymbol{\omega}(\mathbf{i})}(u)\right]_{\mathbf{T}}$.

(ii) Full periodicity: $\left[y_{\mathbf{i}}\left(u+2\left(h+h^{\prime}\right)\right]_{\mathbf{T}}=\left[y_{\mathbf{i}}(u)\right]_{\mathbf{T}}\right.$.

Here, $h$ and $h^{\prime}$ are the Coxeter numbers of $X_{r}$ and $X_{r^{\prime}}^{\prime}$, and $\boldsymbol{\omega}=\omega \times \omega^{\prime}$, where $\omega$ (resp. $\left.\omega^{\prime}\right)$ is the Dynkin automorphism of $X_{r}\left(\right.$ resp. $\left.X_{r^{\prime}}^{\prime}\right)$ for types $A_{r}, D_{r}(r$ odd), or $E_{6}$, and the identity otherwise.

Proof. This is an immediate consequence of the factorization property of the tropical Y-system studied in [Nkn, Proposition 3.2].

As a corollary of Theorems 5.1 and 7.1, we obtain the periodicities of the T-systems and Y-systems.

Corollary 7.2. The following relations hold:

(i) Half periodicity: $T_{\mathbf{i}}\left(u+h+h^{\prime}\right)=T_{\boldsymbol{\omega}(\mathbf{i})}(u), Y_{\mathbf{i}}\left(u+h+h^{\prime}\right)=Y_{\boldsymbol{\omega}(\mathbf{i})}(u)$.

(ii) Full periodicity: $T_{\mathbf{i}}\left(u+2\left(h+h^{\prime}\right)\right)=T_{\mathbf{i}}(u), Y_{\mathbf{i}}\left(u+2\left(h+h^{\prime}\right)\right)=Y_{\mathbf{i}}(u)$.

\section{Acknowledgements}

We thank Pierre-Guy Plamondon for making his result in [Pl1, Pl2] available to us prior to publication.

\section{References}

[A] C. Amiot, Cluster categories for algebras of global dimension 2 and quivers with potential, Ann. Inst. Fourier (Grenoble) 59 (2009), 2525-2590. Zbl 1239.16011 MR 2640929 
[CGT] R. Caracciolo, F. Gliozzi and R. Tateo, A topological invariant of RG flows in 2D integrable quantum field theories, Int. J. Modern Phys. 13 (1999), 2927-2932. Zbl 1229.81194 MR 1719491

[C] F. Chapoton, Functional identities for the Rogers dilogarithm associated to cluster Ysystems, Bull. London Math. Soc. 37 (2005), 755-760. Zbl 1134.33303 MR 2164838

[DK] R. Dehy and B. Keller, On the combinatorics of rigid objects in 2-Calabi-Yau categories, Int. Math. Res. Notices 2008, rnn029, 17 pp. Zbl 1144.18009 MR 2428855

[DWZ1] H. Derksen, J. Weyman and A. Zelevinsky, Quivers with potentials and their representations I: Mutations, Selecta Math. 14 (2008), 59-119. Zbl 1204.16008 MR 2480710

[DWZ2] _ Q Quivers with potentials and their representations II: Applications to cluster algebras, J. Amer. Math. Soc. 23 (2010), 749-790. Zbl 1208.16017 MR 2629987

[DiK] P. Di Francesco and R. Kedem, Q-systems as cluster algebras II: Cartan matrix of finite type and the polynomial property, Lett. Math. Phys. 89 (2009), 183-216. Zbl 1195.81077 MR 2551179

[FZ1] S. Fomin and A. Zelevinsky, Cluster algebras II. Finite type classification, Invent. Math. 154 (2003), 63-121. Zbl 1054.17024 MR 2004457

[FZ2] _ Y-systems and generalized associahedra, Ann. of Math. 158 (2003), 977-1018. Zbl 1057.52003 MR 2031858

[FZ3] Cluster algebras IV. Coefficients, Compos. Math. 143 (2007), 112-164. Zbl 1127.16023 MR 2295199

[FS] E. Frenkel and A. Szenes, Thermodynamic Bethe ansatz and dilogarithm identities. I, Math. Res. Lett. 2 (1995), 677-693. Zbl 1036.11514 MR 1362962

[FK] C. Fu and B. Keller, On cluster algebras with coefficients and 2-Calabi-Yau categories, Trans. Amer. Math. Soc. 362 (2010), 859-895. Zbl 1201.18007 MR 2551509

[GT] F. Gliozzi and R. Tateo, ADE functional dilogarithm identities and integrable models, Phys. Lett. B 348 (1995), 84-88. MR 1325407

[Her1] D. Hernandez, The Kirillov-Reshetikhin conjecture and solutions of T-systems, J. Reine Angew. Math. 596 (2006), 63-87. Zbl 1160.17010 MR 2254805

[Her2] Drinfeld coproduct, quantum fusion tensor category and applications, Proc. London Math. Soc. 95 (2007), 567-608. Zbl 1133.17010 MR 2368277

[HL] D. Hernandez and B. Leclerc, Cluster algebras and quantum affine algebras, Duke Math. J. 154 (2010) 265-341. Zbl pre05788166 MR 2682185

[HW] H. C. Hutchins and H. J. Weinert, Homomorphisms and kernels of semifields, Period. Math. Hungar. 21 (1990), 113-152. Zbl 0718.16041 MR 1070951

[IIKKN] R. Inoue, O. Iyama, B. Keller, A. Kuniba and T. Nakanishi, Periodicities of T-systems and Y-systems, dilogarithm identities, and cluster algebras II: Types $C_{r}, F_{4}$, and $G_{2}$, Publ. RIMS Kyoto Univ. 49 (2013), 43-85.

[IIKNS] R. Inoue, O. Iyama, A. Kuniba, T. Nakanishi and J. Suzuki, Periodicities of T-systems and Y-systems, Nagoya Math. J. 197 (2010), 59-174. Zbl pre05704436 MR 2649278

[Ke1] B. Keller, Cluster algebras, quiver representations and triangulated categories, in Triangulated categories, T. Holm et al. (eds.), London Math. Soc. Lecture Note Ser. 375, Cambridge Univ. Press, 2010, 76-160. Zbl 1215.16012 MR 2681708

[Ke2] _ The periodicity conjecture for pairs of Dynkin diagrams, arXiv:1001.1531.

[KY] B. Keller and D. Yang, Derived equivalences from mutations of quivers with potential, Adv. Math. 226 (2011), 2118-2168. Zbl pre05848698 MR 2739775

[Ki1] A. N. Kirillov, Identities for the Rogers dilogarithm function connected with simple Lie algebras, J. Soviet Math. 47 (1989), 2450-2458. Zbl 0685.33014 MR 0947332 
[Ki2] _ Dilogarithm identities, Progr. Theor. Phys. Suppl. 118 (1995), 61-142. Zbl 0894.11052 MR 1356515

$[\mathrm{Ku}] \quad$ A. Kuniba, Thermodynamics of the $U_{q}\left(X_{r}^{(1)}\right)$ Bethe ansatz system with $q$ a root of unity, Nuclear Phys. B 389 (1993), 209-244. MR 1202213

[KN] A. Kuniba and T. Nakanishi, Spectra in conformal field theories from the Rogers dilogarithm, Modern Phys. Lett. A 7 (1992), 3487-3494. Zbl 1021.81842 MR 1192727

[KNS1] A. Kuniba, T. Nakanishi and J. Suzuki, Characters in conformal field theories from thermodynamic Bethe ansatz, Modern Phys. Lett. A 8 (1993), 1649-1659. Zbl 1021.81843 MR 1229643

[KNS2] _ Functional relations in solvable lattice models: I. Functional relations and representation theory, Int. J. Modern Phys. A 9 (1994), 5215-5266. Zbl 0985.82502 MR 1304819

[KNS3] _ T-systems and Y-systems for quantum affinizations of quantum Kac-Moody algebras, SIGMA 5 (2009), paper 108, 23 pp. Zbl 1201.17009 MR 2591898

[L] L. Lewin, Polylogarithms and associated functions, North-Holland, Amsterdam, 1981. Zbl 0465.33001 MR 0618278

[Nah] W. Nahm, Conformal field theory and torsion elements of the Bloch group, in Frontiers in number theory, physics, and geometry II, Springer, Berlin, 2007, 67-132 Zbl 1193.81092 MR 2290759

[NK] W. Nahm and S. Keegan, Integrable deformations of CFTs and the discrete Hirota equations, arXiv.0905.3776.

[Nkj] H. Nakajima, $t$-analogs of $q$-characters of Kirillov-Reshetikhin modules of quantum affine algebras, Represent. Theory 7 (2003), 259-274. Zbl 1078.17008 MR 1993360

[Nkn] T. Nakanishi, Dilogarithm identities for conformal field theories and cluster algebras: simply laced case, Nagoya Math. J. 202 (2011), 23-43. Zbl pre05920542 MR 2804544

[Pa] Y. Palu, Cluster characters for 2-Calabi-Yau triangulated categories, Ann. Inst. Fourier (Grenoble) 58 (2008), 2221-2248. Zbl 1154.16008 MR 2473635

[Pl1] P.-G. Plamondon, Cluster characters for cluster categories with infinite-dimensional morphism spaces, Adv. Math. 227 (2011), 1-39. Zbl pre05880817 MR 2782186

[P12] C Cluster algebras via cluster categories with infinite-dimensional morphism spaces, Compos. Math. 147 (2011), 1921-1954. Zbl 1244.13017 MR 2862067

[Zag] D. Zagier, The dilogarithm function, in Frontiers in number theory, physics, and geometry II, Springer, Berlin, 2007, 3-65. Zbl 1176.11026 MR 2290758 